\title{
HiMod Reduction of Advection-Diffusion-Reaction Problems with General Boundary Conditions
}

\author{
Matteo C. Aletti ${ }^{1}$ - Simona Perotto ${ }^{2}$ (D) . \\ Alessandro Veneziani ${ }^{3,4}$
}

\begin{abstract}
We extend the hierarchical model reduction procedure previously introduced in Ern et al. (in: Kunisch, Of, Steinbach (eds) Numerical mathematics and advanced applications, Springer, Berlin, pp 703-710, 2008) and Perotto et al. (Multiscale Model Simul $8(4): 1102-1127,2010)$ to deal with general boundary conditions, enforcing their prescription in the basis function set. This is achieved by solving a Sturm-Liouville Eigenvalue problem. We analyze this approach and provide a convergence analysis for the associated error in the case of a linear advection-diffusion-reaction problem in rectangles (2D) and slabs (3D). Numerical results confirm the theoretical investigation and the reliability of the proposed approach.
\end{abstract}

Keywords Model reduction $\cdot$ Spectral/finite element combined approximation $\cdot$ Robin boundary conditions $\cdot$ Sturm-Liouville Eigenvalue problem

Mathematics Subject Classification 65N30 $\cdot 65$ N35 $\cdot 76 \mathrm{M} 10 \cdot 76 \mathrm{M} 22 \cdot 78 \mathrm{M} 34$

Simona Perotto

simona.perotto@polimi.it

Matteo C. Aletti

matteo.aletti@inria.fr

Alessandro Veneziani

avenez2@emory.edu

1 Laboratoire Jacques-Louis Lions, Inria Paris, Sorbonne Universités UPMC Univ Paris 6, Paris, France

2 MOX, Dipartimento di Matematica, Politecnico di Milano, P.zza L. da Vinci 32, 20133 Milan, Italy

3 Department of Mathematics and Computer Science, Emory University, 400 Dowman Dr NE, S.te N416, Atlanta, GA 30322, USA

4 School of Advanced Studies IUSS, P.zza della Vittoria 15, 27100 Pavia, Italy

This is a post-peer-review, pre-copyedit version of an article published in J Sci Compu,, 2018, 76, 89-119.

The final authenticated version is available online at: https://doi.org/10.1007/s10915-017-0614-5 


\section{Introduction}

Efficient numerical solution of problems characterized by a main direction like flow in pipes and networks has been investigated in several ways, as witnessed by engineering and math-ematical literature (see, e.g., $[15,16,24,25])$. A popular approach is based on reducing the problem of interest to a one-dimensional setting along the mainstream, after dropping trans-verse dynamics. The one-dimensional Euler equations in gas- and haemo-dynamics are a popular example. A different numerical approach known as hierarchical model (HiMod) reduction, yielding fast computation without discarding the transverse dynamics, has been proposed in [14,27], and successively investigated in [26,29-31]. In particular, the HiMod procedure follows the idea of combining separation of variables with a diverse numerical approximation, to take advantage from particular features of the problem at hand. Thus, along the mainstream we consider a classical one-dimensional finite element approximation to exploit easiness and versatility of this method. The transverse components are tackled by different approximations. In many applications of fluid dynamics, the cross-section of pipes is regular enough to accommodate a spectral approach $[3,10-12,19,36-38]$. The rapid con-vergence of spectral approximations allows to capture the important features of the transverse dynamics with a relatively low number of modes. This results in accurate approximations with a lower number of degrees of freedom in comparison with non-customized discretizations like classical finite elements. The separation of variables performed by a HiMod reduction carries several advantages also for the general structure of the algebraic problems, for model adaptivity [29] and for the efficiency of the solver. This has been recently demonstrated for the incompressible Navier-Stokes equations in biomedical applications in [17,22], both in terms of accuracy and computational efficiency.

One of the most significant limitations of the HiMod approach pursued in all the papers mentioned above is represented by the boundary conditions to prescribe on the lateral bound-ary of the domain. Homogeneous Dirichlet conditions were promptly included by the basis functions adopted for this problem (either sinusoidal or polynomial). Although these condi-tions describe many practical applications, in view of generalizing the HiMod approach to more complex problems such as fluid-structure interaction, we need to address more general boundary conditions. We present here a possible method for this, based on the solution of an appropriate Sturm-Liouville Eigenvalue (SLE) problem [9]. This allows to design the modal basis to include, in an essential way, general boundary conditions and some features of the problem to solve, so that we call this an educated basis, while referring to the associated HiMod formulation as to $e$-HiMod reduction. The SLE problems in the HiMod construc-tion of modal functions represents the main novelty of this work. We explore the theoretical aspects of this together with some preliminary (yet nontrivial) numerical test cases. As this is a work in progress, we will explore more practical applications elsewhere-following the line of [22].

The paper is organized as follows. Section 2 recalls some basics of HiMod reduction. Section 3 introduces the basic properties of SLE problem, and explicitly provide some proofs for general boundary conditions, extending the standard literature focused on Dirichlet boundary data. In Sect. 4 we introduce the e-HiMod methodology in either twodimensional rectangu-lar domains or three-dimensional slabs, together with an a priori analysis of the associated model reduction error consequent to the analysis of the previous section. Numerical results confirming theoretical findings are presented both in twodimensional and three-dimensional domains in Sect. 5, dealing with linear advectiondiffusion-reaction problems. Limitations and future developments are summarized in Sect. 6. 


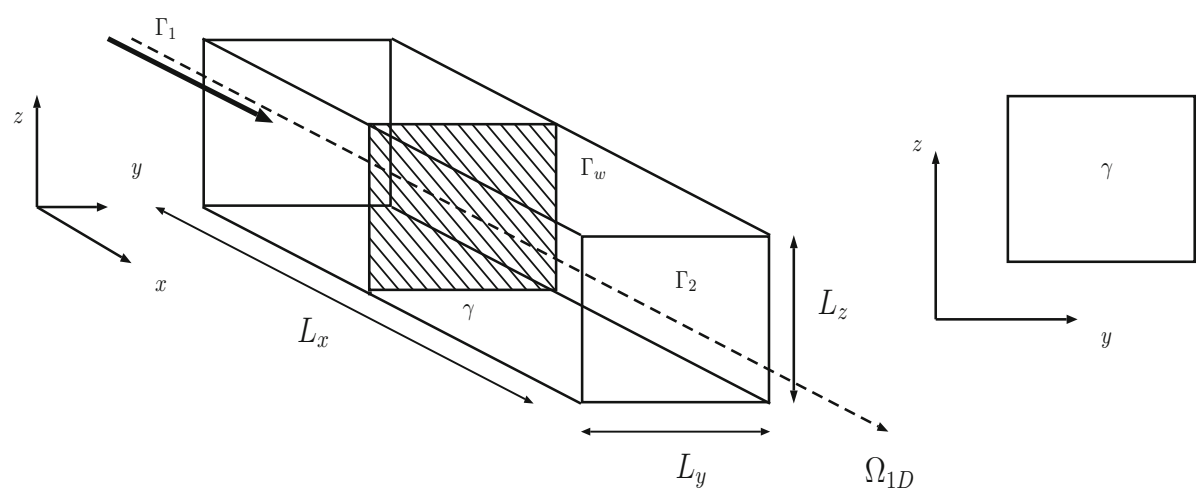

Fig. 1 3D slab domain $\Omega$ (left) and transverse fiber $\gamma($ right)

\section{The HiMod Approach: Basics}

Since in this paper we are concerned with rectangular/slab domains, we directly assume that $\Omega \subset \mathbb{R}^{d}(d=2,3)$ coincides with the Cartesian product $\Omega_{1 D} \times \gamma$, where $\Omega_{1 D}=[0, L]$ is the supporting domain and $\gamma=(0,1)^{d-1}$ is the transverse fiber (see Fig. 1). While this is the domain we will consider in presenting the method, it is important to stress that the entire procedure can be extended to more complex geometries. In the latter case, the domain considered here has the role of a reference geometry, as done, for instance, in a Dirichlet boundary condition setting in [22]. The space coordinates on $\gamma$ will be denoted by $\mathbf{y}$. The axial direction associated with $\Omega_{1 D}$ is dominant with respect to the others (i.e., $L \gg 1$ ). In general, we may assume $\Omega_{1 D}$ to be a curve $\mathcal{C}:(0, L) \rightarrow \Omega_{1 D} \subset \mathbb{R}^{2}$, where $x$ denotes a curvilinear abscissa $[26,28]$, while fiber $\gamma$ is replaced by a fiber $\gamma_{x}$ coinciding with a regular function of $x$.

In $\Omega$ we solve the standard scalar linear advection-diffusion-reaction (ADR) problem completed with (homogeneous) generic boundary conditions,

$$
\mathcal{L} u=f \quad \text { in } \Omega, \quad \mathcal{B} u=0 \text { on } \partial \Omega,
$$

where $\mathcal{L}: V \rightarrow V^{\prime}$ stands for the differential operator $\mathcal{L} u=-\nabla \cdot(\mu \nabla u)+\nabla \cdot(\boldsymbol{\beta} u)+\sigma u$, from the Hilbert space $V \subset H^{1}(\Omega)$ (whose definition depends on the boundary conditions) to its dual $V^{\prime}$, and $\mathcal{B} u=0$ denotes generically standard boundary conditions (Dirichlet, Neumann, Robin), possibly coexistent on different portions of $\partial \Omega$. With standard arguments (and notation) [21], we associate with this problem the bilinear form $a(u, v)$ on $V \times V$ and the functional $F(v)$ with arguments in $V$, as follows

$$
a(u, v)=\int_{\Omega}[\mu \nabla u \cdot \nabla v+(\nabla \cdot(\boldsymbol{\beta} u)+\sigma u) v] d \Omega, \quad F(v)=\int_{\Omega} f v d \Omega .
$$

We postulate the boundary $\partial \Omega$ to consist of the two transverse fibers $\Gamma_{1}=\{0\} \times \gamma$ and $\Gamma_{2}=\{L\} \times \gamma$, and of the lateral boundary $\Gamma_{w}=\partial \Omega \backslash\left\{\Gamma_{1} \cup \Gamma_{2}\right\}$ (see Fig. 1). The present paper focuses on the treatment of generic boundary conditions on $\Gamma_{w}$, while the introduction of generic conditions on $\Gamma_{1}$ and $\Gamma_{2}$ does not rise problems [27]. For simplicity, but with no loss of generality, we assume there Dirichlet homogeneous data. In addition, we assume the diffusivity coefficient $\mu \in L^{\infty}(\Omega)$, with $\mu \geq \mu_{0}>0$ a.e. in $\Omega$, the convective field $\boldsymbol{\beta} \in\left[L^{\infty}(\Omega)\right]^{d}$, the reactive coefficient $\sigma \in L^{\infty}(\Omega)$, and the forcing term $f \in L^{2}(\Omega)$. We 
also assume that $\nabla \cdot \boldsymbol{\beta} \in L^{\infty}(\Omega)$ and that $-\frac{1}{2} \nabla \cdot \boldsymbol{\beta}+\sigma>0$ a.e. in $\Omega$, so that the bilinear form is coercive and the well-posedness of the weak form of (1) follows from the Lax-Milgram lemma. Correspondingly to the boundary conditions in (1), we denote by $V=H_{*}^{1}(\Omega)$ the subspace of $H^{1}(\Omega)$ functions such that $\mathcal{B} u=0$ on $\partial \Omega$, the boundary conditions being imposed in an essential way, regardless of the specific type.

The HiMod formulation requires a specific function setting. We introduce the onedimensional space $V_{1 D}=H_{0}^{1}\left(\Omega_{1 D}\right)$ associated with the supporting fiber. On the transverse direction, we consider a set of modal functions $\left\{\varphi_{k}\right\}_{k}$, defined on $\gamma$ such that $\mathcal{B} \varphi_{k}=0$, and set

$$
V_{\gamma, m}=\operatorname{span}\left(\left\{\varphi_{k}\right\}_{k=1}^{m}\right)
$$

Thus, the hierarchically reduced semi-discrete space is given by

$$
V_{m}=\left\{v(x, \mathbf{y})=\sum_{k=1}^{m} v_{k}(x) \varphi_{k}(\mathbf{y}), \text { with } v_{k} \in V_{1 D} \text { for } k=1 \ldots m\right\} .
$$

We assume [14,27] that $V_{m} \subset V$ for any $m \in \mathbb{N}$ (conformity hypothesis), and that, for any $v \in V, \lim _{m \rightarrow+\infty}\left(\inf _{v_{m} \in V_{m}}\left\|v-v_{m}\right\|_{V}\right)=0$ (spectral approximability hypothesis). For $m \rightarrow \infty$, the conformity and spectral approximability properties postulated on $V_{m}$ lead to a space $V_{\infty}$ dense in $V$. The modal index $m$ can be selected either a priori or adaptively with an a posteriori modeling error analysis. In this paper we adopt the first strategy, while referring the interested reader to [29,31] for an example of adaptive HiMod reduction in both steady and unsteady settings, respectively.

The basis functions $\left\{\varphi_{k}\right\}_{k}$ do not need to be generally orthonormal. However, if we assume they are orthonormal with respect to the $L^{2}(\gamma)$-scalar product, $(\cdot, \cdot)_{\gamma}$, the coefficients $v_{k}$ in (3) coincide with the standard Fourier coefficients $v_{k}=\left(v, \varphi_{k}\right)_{\gamma}$. Modal functions $\left\{\varphi_{k}\right\}_{k}$ can be selected a priori, being, for instance, trigonometric functions, Legendre polynomials or B-splines [8, 14, 17, 22,26-28].

The fully-discrete HiMod formulation is obtained by introducing a uniform subdivision $\mathcal{T}_{h}$ of step $h$ along $\Omega_{1 D}$, with the nodes $x_{i}, i=0,1, \ldots, N_{h}$. The generalization to the case of non-uniform adapted partitions is considered in [29]. Let $V^{h}$ be the subspace of $V_{1 D}$ of the continuous piecewise linear functions associated with $\mathcal{T}_{h}$ and vanishing at $x_{0}=0$ and $x_{N_{h}}=L$. Higher order discretizations can be considered as well [28]. Correspondingly, we define the space $V_{m}^{h}$ of functions in the form $\sum_{k=1}^{m} v_{k}^{h}(x) \varphi_{k}(\mathbf{y})$, with $v_{k}^{h} \in V^{h}$. Let $\psi_{i}$ denote the Lagrangian basis function in $V^{h}$ associated with the node $x_{i}$. Thus, we can consider the discrete modal representation

$$
u_{m}^{h}(x, \mathbf{y})=\sum_{k=1}^{m} u_{k}^{h}(x) \varphi_{k}(\mathbf{y})=\sum_{k=1}^{m} \sum_{i=1}^{N_{h}} u_{k, i} \psi_{i}(x) \varphi_{k}(\mathbf{y}),
$$

where $u_{k, i}$ are the actual unknowns of the discrete HiMod formulation

$$
\text { find } u_{m}^{h} \in V_{m}^{h}: a\left(u_{m}^{h}, v^{m^{h}}\right)=F\left(v^{h}\right) \quad \forall v^{h} \in V_{m}^{h}
$$

with $u_{k}^{h}(x)=\sum_{i=1}^{N_{h}} u_{k, i} \psi_{i}(x) \in V^{h}$. A natural choice for the test function in (5) is $v_{m}^{h}(x, \mathbf{y})=\psi_{l}(x) \varphi_{j}(\mathbf{y})$, with $l=1, \ldots, N_{h}, j=1, \ldots, m$. Then, the HiMod formulation (5) reduces to: find $u_{k, i} \in \mathbb{R}$, with $k=1, \ldots, m$ and $i=1, \ldots, N_{h}$, such that, for any 
$j=1, \ldots, m$ and for any $l=1, \ldots, N_{h}$,

$$
\begin{aligned}
& \sum_{k=1}^{m} \sum_{i=1}^{N_{h}}\left\{\int _ { \Omega _ { 1 D } } \left[r_{k, j}^{11}(x) \frac{\partial \psi_{i}}{\partial x}(x) \frac{\partial \psi_{l}}{\partial x}(x)+r_{k, j}^{10}(x) \frac{\partial \psi_{i}}{\partial x}(x) \psi_{l}(x)\right.\right. \\
& \left.\left.\quad+r_{k, j}^{00} \psi_{i}(x) \psi_{l}(x)\right] d x\right\} u_{k, i}=\int_{\Omega_{1 D}} \psi_{l}(x) f_{j}(x) d x .
\end{aligned}
$$

Following [14], in the case of homogeneous Dirichlet data also on $\Gamma_{w}$, coefficients $r_{k, j}^{s t}$, with $s, t=0,1$, and $f_{j}$ are given by

$$
\begin{aligned}
r_{k, j}^{11}(x) & =\int_{\gamma} \mu(x, \mathbf{y}) \varphi_{j}(\mathbf{y}) \varphi_{k}(\mathbf{y}) d \mathbf{y}, \quad r_{k, j}^{10}(x)=\int_{\gamma} \beta_{1}(x, \mathbf{y}) \varphi_{j}(\mathbf{y}) \varphi_{k}(\mathbf{y}) d \mathbf{y} \\
r_{k, j}^{00}(x) & =\int_{\gamma}\left(\mu(x, \mathbf{y}) \varphi_{j}^{\prime}(\mathbf{y}) \varphi_{k}^{\prime}(\mathbf{y})+\beta_{2}(x, \mathbf{y}) \varphi_{j}^{\prime}(\mathbf{y}) \varphi_{k}(\mathbf{y})+\sigma(x, \mathbf{y}) \varphi_{j}(\mathbf{y}) \varphi_{k}(\mathbf{y})\right) d \mathbf{y} \\
f_{j}(x) & =\int_{\gamma} f(x, \mathbf{y}) \varphi_{j}(\mathbf{y}) d \mathbf{y}
\end{aligned}
$$

respectively, where $\varphi_{l}^{\prime}$ denotes the derivative of the generic modal function $\varphi_{l}$ with respect to $\mathbf{y}$. In particular, coefficients $r_{k, j}^{s t}(s, t=0,1)$ account for the transverse dynamics after the reduction phase. From an algebraic viewpoint, discretization (6) leads to a system of $m$ coupled one-dimensional problems, characterized by a block tridiagonal sparsity pattern. Devising special linear algebra solvers and preconditioners that exploit this special pattern is subject of active research [5], and it is expected to bring significant efficiency of the solution step. As for now, it is fair comparing the number of degrees of freedom (dof), noting that HiMod scales as $m^{d-1} N_{h}$ that favorably compares with $N_{h}^{d}$ dof of a standard finite element approximation, for $m \ll N_{h}$. This is the expected advantage of the separation of variables in the specific skewed or pipe-like domains of interest. With appropriate preconditioners, we expect the cost of each HiMod solve to be $\mathcal{O}\left(m^{2 d-1} N_{h}^{2}\right)$, so that adding $n$ modes on each transverse direction will add a relative cost of the order of $\mathcal{O}\left((m+n)^{2 d-1} N_{h}^{2} / m^{2 d-1} N_{h}^{2}\right)$ $\approx 1+2 n / m$.

The convergence of the HiMod discrete solution $u_{m}^{h}$ to the continuous one $u$ for $m \rightarrow+\infty$ and $h \rightarrow 0$ in presence of Dirichlet boundary conditions on $\Gamma_{w}$ is stated in Proposition 3.1 of [27]. For instance, in Fig. 2 we show the results of a test case on a slab, carried out with the open source library LifeV. ${ }^{1}$ Specifically, we provide the results of a constant coefficient ADR problem characterized by a forcing term featuring two Gaussian functions centered at two distinct points. Homogeneous Dirichlet conditions are prescribed on $\Gamma_{w}$ and $\Gamma_{1}$, while homogeneous Neumann data are assigned on $\Gamma_{2}$. The contour plots highlight the agreement of the HiMod approximation with the finite element solution, progressively improving with $m$. The finite element solution is obtained with a piecewise linear approximation on a uniform grid $\left(h_{x}=0.1, h_{y}=h_{z}=h_{x} / 2\right)$. The HiMod approximation is yielded on the same one-dimensional grid along the $x$-axis and with an increasing number of modes. Figure 3 provides more quantitative information about this comparison. In particular, it highlights how the HiMod solution is consistently more accurate with respect to the full 3D finite element approximation for a fixed number of degrees freedom (left panel), and requires a considerably lower computational time for a desired error (right panel). More details on this test case can be found in [1].

\footnotetext{
1 LifeV is an open source finite element library developed by MOX at Politecnico di Milano, Italy, the Department of Mathematics at EPFL, Switzerland and the Department of Mathematics and Computer Science at Emory University, USA (https://cmcsforge.epfl.ch/doxygen/lifev/).
} 


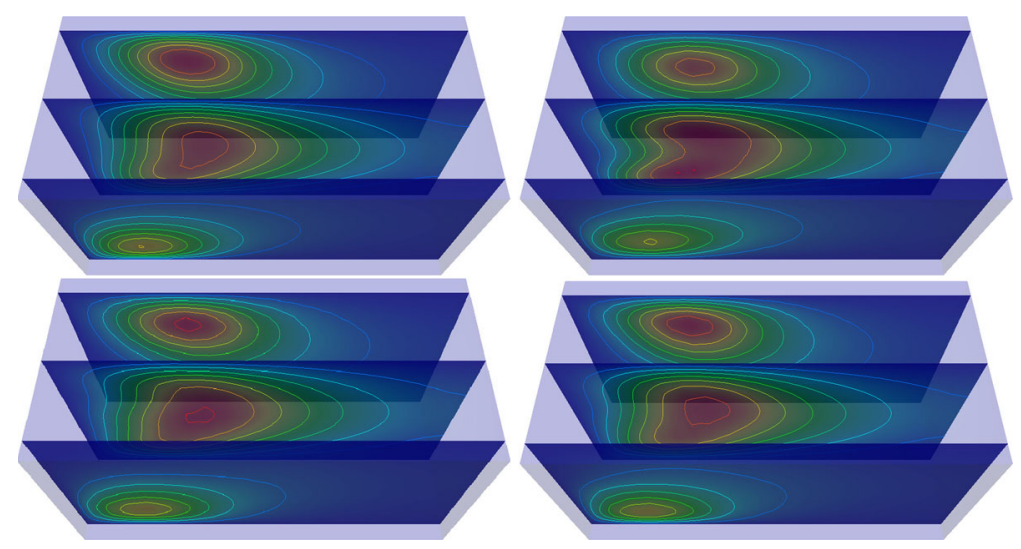

Fig. 2 3D ADR problem: longitudinal sections of the FE solution (top-left) and of the HiMod solutions for $m=9$ (top-right), $m=16$ (bottom-left) and $m=25$ (bottom-right)

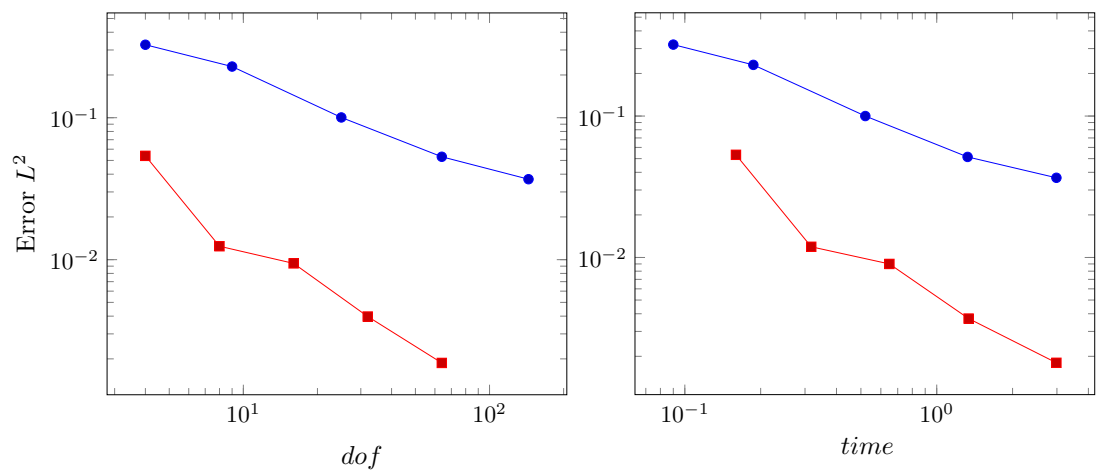

Fig. 3 3D ADR problem with lateral Dirichlet boundary conditions: $L^{2}(\Omega)$-norm of the global error as a function of the number of dof (left) and of the computational time (s) (right) for a standard 3D linear finite element discretization (circle markers) and for a HiMod approximation (square markers)

\section{The SLE Problem in the HiMod Framework}

Directional model reduction has been considered in different contexts. In particular, in [36] the reduction is oriented to shell structure problems and the optimal selection of the reduced basis is functional to the small size of the transverse dimension. In the present work we do not analyze the modeling error as a function of the transverse size of the domain. We construct our discussion purely on the spectral approximation properties of the basis functions. The different possible choices of the modal basis for Dirichlet boundary conditions are discussed in $[14,22,23,27]$, yet the case of general conditions needs to be carefully addressed and analyzed. This is exactly the purpose of the present work.

The construction of our modal basis relies on the eigenfunctions of an appropriate differential operator designed on the problem to solve. In particular, when considering the template problem (1), we search a basis function set incorporating the lateral boundary conditions in an essential way. Let $\mathcal{L}_{S}: V \rightarrow L^{2}(\Omega)$ be a symmetric operator. For instance, $\mathcal{L}_{S}$ may be 
the Laplacian or the symmetric part of the ADR operator in (1), namely

$$
\mathcal{L}_{S} u=-\nabla \cdot(\mu \nabla u)+\widetilde{\sigma} u,
$$

with $\tilde{\sigma}=\sigma+\frac{1}{2} \nabla \cdot \boldsymbol{\beta}$. Since there is no ambiguity in what follows, we adopt the same symbol to denote the restriction of this operator to the $d-1$-dimensional fiber $\gamma$. Then, the computation of a HiMod basis for problem (1) is obtained by solving the eigenvalue problem

$$
\mathcal{L}_{S} \varphi=\lambda \varphi \quad \text { in } \gamma, \quad \mathcal{B} \varphi=0 \text { on } \partial \gamma .
$$

We postulate the problem coefficients to be regular enough for the operator $\mathcal{L}_{S}$ to be self-adjoint. Problem (9) is a classical Sturm Liouville Eigenvalue (SLE) problem, largely investigated in the literature of partial differential equations as a natural tool for analytically solving simple problems by separation of variables/superposition of effects $[8,9,32]$. We recall here the basic properties of SLE problems and provide proofs in a general context, as many references are mostly concerned with one-dimensional problems completed with Dirichlet boundary conditions.

\subsection{Spectrum of a Self-adjoint Elliptic Operator}

We consider the SLE problem with the general form

$$
\mathcal{L}_{S} \varphi_{k}(\mathbf{y})=\lambda_{k} w(\mathbf{y}) \varphi_{k}(\mathbf{y}) \text { in } \gamma, \quad \mathcal{B} \varphi_{k}(\mathbf{y})=0 \text { on } \partial \gamma
$$

where $\lambda_{k}$ is the eigenvalue of $\mathcal{L}_{S}$ associated with the eigenfunction $\varphi_{k}$, while the weight $w$ is a positive continuous function. The following statements hold:

1. the eigenvalues $\left\{\lambda_{k}\right\}_{k}$ are real and form a countable monotone non-decreasing sequence convergent to infinity for $k \rightarrow+\infty$. In the one-dimensional case, the multiplicity of every eigenvalue is equal to one (it may be greater for singular SLE problems, where $\mu$ is allowed to vanish on the boundary);

2. the eigenfunctions $\left\{\varphi_{k}\right\}_{k}$ are orthonormal with respect to the scalar product of the weighted space $L_{w}^{2}(\gamma)$. They constitute a complete set in the same space. This means that, for a generic function $f \in L_{w}^{2}(\gamma)$, the truncated series

$$
\mathcal{S}_{m} f(\mathbf{y})=\sum_{k=1}^{m} \hat{f}_{k} \varphi_{k}(\mathbf{y}),
$$

with $\hat{f}_{k}=\int_{\gamma} w(\mathbf{y}) f(\mathbf{y}) \varphi_{k}(\mathbf{y}) d \mathbf{y}$, is such that $\lim _{m \rightarrow+\infty}\left\|f-\mathcal{S}_{m} f\right\|_{w}=0$, with $\|\cdot\|_{\omega}$ the norm associated with the space $L_{w}^{2}(\gamma)$. From now on, we refer to the basis functions $\left\{\varphi_{k}\right\}_{k}$ in (10) guaranteeing expansion (11) as to the SL basis;

3. for $k \rightarrow+\infty$, the eigenvalues in (10) are such that

$$
\lambda_{k} \sim \mathcal{O}\left(\frac{k^{\frac{2}{d-1}}}{|\gamma|}\right),
$$

with $|\gamma|$ the size of the fiber $\gamma$. Result (12) follows from the so-called Weyl formula $[32,39]$. 


\subsection{Approximability Properties}

Let $\mathcal{R}_{m} f$ denote the residual associated with the $m$-th truncated series (11), namely

$$
\mathcal{R}_{m} f(\mathbf{y})=f(\mathbf{y})-\mathcal{S}_{m} f(\mathbf{y})=\sum_{k=m+1}^{+\infty} \hat{f}_{k} \varphi_{k}(\mathbf{y}) .
$$

We will investigate the convergence rate of the residual with respect to $m$ on the domain $\gamma$. To this aim, we first establish the dependence of the generalized Fourier coefficient $\hat{f}_{k}$ on the eigenvalue $\lambda_{k}$. In particular, when function $f$ belongs at least to $H^{2}(\gamma)$, we can compute also the generalized Fourier series of $\mathcal{L}_{S} f$, whose $k$-th coefficient will be denoted by $\widehat{\mathcal{L}_{S} f}$.

Lemma 1 Let $\varphi_{k}$ be the eigenfunction solution to problem (10), and let $f \in H^{2}(\gamma)$ be a generic function fulfilling the same boundary conditions as $\varphi_{k}$. Then,

$$
\hat{f}_{k}=\frac{1}{\lambda_{k}} \widehat{\mathcal{L}}_{S} f_{k} \quad \forall k \geq 1 .
$$

Moreover, if $f \in H^{2 p}(\gamma)$, with $p \geq 2$, and $\mathcal{L}_{S}^{r} f$ satisfies the same boundary conditions as $f$ for any $r \leq p-1$ (i.e., $\mathcal{L}_{S}^{r} f$ satisfies what we refer to as compatible boundary conditions up to order $p-1)$, then

$$
\hat{f}_{k}=\left(\frac{1}{\lambda_{k}}\right)^{p} \widehat{\mathcal{L}}_{S}^{p} f_{k} \quad \forall k \geq 1,
$$

where $\widehat{\mathcal{L}_{S}^{p} f_{k}}$ denotes the $k$-th coefficient of the generalized Fourier series associated with the p-th power of the operator $\mathcal{L}_{S}$.

Proof Statement (13) follows from (10) and from the fact that $\mathcal{L}_{S}$ is a self-adjoint operator in $L_{w}^{2}(\gamma)$, so that,

$$
\hat{f}_{k}=\left(f, \varphi_{k}\right)_{L_{w}^{2}(\gamma)}=\frac{1}{\lambda_{k}}\left(f, \mathcal{L}_{S} \varphi_{k}\right)_{L_{w}^{2}(\gamma)}=\frac{1}{\lambda_{k}}\left(\mathcal{L}_{S} f, \varphi_{k}\right)_{L_{w}^{2}(\gamma)}=\frac{1}{\lambda_{k}} \widehat{\mathcal{L}}_{S} f_{k} .
$$

Now, if we consider a function $f \in H^{2 p}(\gamma)$ for some $p \geq 2$ and such that $\mathcal{L}_{S}^{r} f$ fulfills the same boundary conditions as $f$ for $r \leq p-1$, we can iterate the same argument as in (15), to obtain

$$
\widehat{\mathcal{L}}_{S}^{r} f_{k}=\frac{1}{\lambda_{k}} \widehat{\mathcal{L}}_{S}^{r+1} f_{k}
$$

By properly combining (15) with (16), we obtain (14).

From now on, for simplicity, we set $w=1$, as this is the only case we actually considered in the numerical assessment. The convergence of $\mathcal{R}_{m} f$ as a function of $m$ is stated in the following result.

Theorem 1 Let $\varphi_{k}$ be the eigenfunction, solution to problem (10), and let $f \in H^{2}(\gamma)$ satisfy the same boundary conditions as $\varphi_{k}$. Then, there exists a constant $C_{1, s}$ independent of $m$, such that

$$
\left\|\mathcal{R}_{m} f\right\|_{H^{s}(\gamma)} \leq C_{1, s}\left(\frac{1}{m+1}\right)^{\frac{2-s}{d-1}}\|f\|_{H^{2}(\gamma)},
$$

for $s=0,1$, and with $H^{0}(\gamma)=L^{2}(\gamma)$. Moreover, if $f \in H^{2 p}(\gamma)$, with $p \geq 2$, and $\mathcal{L}_{S}^{r}$ fsatisfies compatible boundary conditions up to order $p-1$, then there exists a constant $C_{2, s}$, 
independent of $m$, such that, for $s=0,1$,

$$
\left\|\mathcal{R}_{m} f\right\|_{H^{s}(\gamma)} \leq C_{2, s}\left(\frac{1}{m+1}\right)^{\frac{2 p-s}{d-1}}\|f\|_{H^{2 p}(\gamma)} .
$$

Proof We first consider the case $s=0$. By resorting to Parseval's identity, we have

$$
\left\|\mathcal{R}_{m} f\right\|_{L^{2}(\gamma)}^{2}=\sum_{k=m+1}^{+\infty} \hat{f}_{k}^{2} .
$$

The properties of the SLE problem listed above together with relation (13) guarantee that the right-hand side converges to zero for $k \rightarrow \infty$. In addition, the slowest term to converge is the one associated with $k=m+1$ since coefficients $\hat{f}_{k}$ inversely depend on $\lambda_{k}$. Thanks to Lemma 1, formula (12) and the $L^{2}(\gamma)$-orthonormality of functions $\left\{\varphi_{k}\right\}_{k}$, we have that, if $f \in H^{2}(\gamma)$ and satisfies the same boundary conditions as $\varphi_{k}$, then

$$
\left\|\mathcal{R}_{m} f\right\|_{L^{2}(\gamma)}^{2} \leq\left(\frac{1}{\lambda_{m+1}}\right)^{2} \sum_{k=m+1}^{+\infty}\left[\widehat{\mathcal{L}_{S} f}\right]^{2} \leq \frac{C}{(m+1)^{\frac{4}{d-1}}}\|f\|_{H^{2}(\gamma)}^{2},
$$

with $C$ depending on $|\gamma|$ and on the parameters of the operator $\mathcal{L}_{S}$, e.g. $\mu, \boldsymbol{\beta}$ and $\sigma$. Analogously, if $f \in H^{2 p}(\gamma)$ for some $p \geq 2$, and $\mathcal{L}_{S}^{r} f$ satisfies the same boundary conditions as $f$ for any $r \leq p-1$, we have

$$
\left\|\mathcal{R}_{m} f\right\|_{L^{2}(\gamma)}^{2} \leq\left(\frac{1}{\lambda_{m+1}}\right)^{2 p} \sum_{k=m+1}^{+\infty}\left[\widehat{\mathcal{L}_{S}^{p} f}\right]^{2} \leq \frac{C}{(m+1)^{\frac{4 p}{d-1}}}\|f\|_{H^{2 p}(\gamma)}^{2} .
$$

Now, we select $s=1$. Let $a_{S}(u, v)$ denote the symmetric coercive bilinear form associated with $\mathcal{L}_{S}$. For instance, when $\mathcal{L}_{S}$ is the symmetric part of the ADR operator, $a_{S}\left(\varphi_{k}, v\right)=$ $\int_{\gamma}\left[\mu \nabla \varphi_{k} \cdot \nabla v+\tilde{\sigma} \varphi_{k} v\right] d \gamma$, defined in $V_{\gamma}=H_{*}^{1}(\gamma)$ the subspace of $H^{1}(\gamma)$-functions satisfying the assigned boundary conditions in an essential way. This form induces the scalar product $((w, v))=a_{S}(w, v)$ for any $w, v \in V_{\gamma}$, and the associated norm $\|w\|_{a_{S}}^{2}=a_{S}(w, w)$ for any $w \in V_{\gamma}$. In particular, the functions $\left\{\widetilde{\varphi}_{k}=\frac{\varphi_{k}}{\sqrt{\lambda_{k}}}\right\}_{k}$ form an orthonormal basis in $H^{1}(\gamma)$ with respect to the scalar product $((\cdot, \cdot))$ [34]. The generalized Fourier coefficients $\widetilde{f}_{k}$ of $f$ with respect to this basis are related to the coefficients in (11) simply by integration by parts, since

$$
\tilde{f}_{k}=\left(\left(f, \widetilde{\varphi}_{k}\right)\right)=a_{S}\left(f, \widetilde{\varphi}_{k}\right)=\lambda_{k}\left(f, \widetilde{\varphi}_{k}\right)=\sqrt{\lambda_{k}}\left(f, \varphi_{k}\right)=\sqrt{\lambda_{k}} \hat{f}_{k}
$$

Via Parseval's identity and thanks to the coercivity of the bilinear form $a_{S}(\cdot, \cdot)$, we obtain

$$
\alpha_{S}\left\|\mathcal{R}_{m} f\right\|_{H^{1}(\gamma)}^{2} \leq\left\|\mathcal{R}_{m} f\right\|_{a_{S}}^{2}=\sum_{k=m+1}^{+\infty} \tilde{f}_{k}^{2}=\sum_{k=m+1}^{+\infty} \lambda_{k} \hat{f}_{k}^{2},
$$

with $\alpha_{S}$ the coercivity constant associated with $a_{S}(\cdot, \cdot)$. The same arguments adopted in (18) and (19) lead to the estimate

$$
\left\|\mathcal{R}_{m} f\right\|_{H^{1}(\gamma)}^{2} \leq \frac{\alpha_{S}^{-1}}{\lambda_{m+1}} \sum_{k=m+1}^{+\infty}\left[{\widehat{\mathcal{L}_{S} f}}_{k}\right]^{2} \leq \frac{C}{(m+1)^{\frac{2}{d-1}}}\|f\|_{H^{2}(\gamma)}^{2},
$$


and

$$
\left\|\mathcal{R}_{m} f\right\|_{H^{1}(\gamma)}^{2} \leq \alpha_{S}^{-1}\left(\frac{1}{\lambda_{m+1}}\right)^{2 p-1} \sum_{k=m+1}^{+\infty}\left[\widehat{\mathcal{L}_{S}^{p} f_{k}}\right]^{2} \leq \frac{C}{(m+1)^{\frac{4 p-2}{d-1}}}\|f\|_{H^{2 p}(\gamma)}^{2},
$$

respectively, where $C$ now depends also on the coercivity constant $\alpha_{S}$.

Remark 1 For $p \rightarrow \infty$, estimate (17) yields spectral convergence. In the specific case of Neumann conditions, this means that an infinitely regular function $f$ with all the odd derivatives vanishing at the boundary is spectrally approximated by generalized Fourier truncated series. This result is recalled, for instance, in Section 2.2 of [9] (with no proof.)

\subsubsection{The Case of Neumann Boundary Conditions}

Driven by numerical evidence, for the case with Neumann boundary conditions, we can prove an additional result under some regularity assumptions that, however, do not involve the boundary conditions for the derivatives of $f$ (as opposed to Theorem 1). At the best of authors' knowledge, this result is non standard.

When $\mathcal{L}_{S}$ is the symmetric part of the ADR operator, we assume the coefficients $\mu, \boldsymbol{\beta}$ and $\sigma$ in (8) to be regular enough to make each step formally correct.

Lemma 2 Let $\varphi_{k}$ be the eigenfunction, solution to problem (10) completed with homogeneous Neumann boundary conditions. If $f \in H^{4}(\gamma)$ and satisfies homogeneous Neumann boundary conditions as $\varphi_{k}$, then we have

$$
\left|\hat{f}_{k}\right| \leq C \frac{1}{\lambda_{k}^{3 / 2}}\|f\|_{H^{4}(\gamma)} \quad \forall k \geq 1
$$

with $C=C(\tau), \tau$ being the constant associated with the trace inequality. In addition, if the basis functions $\left\{\varphi_{k}\right\}_{k}$ are uniformly bounded with respect to $k$, then the previous statement improves into

$$
\left|\hat{f}_{k}\right| \leq C \frac{1}{\lambda_{k}^{2}}\|f\|_{H^{4}(\gamma)} \quad \forall k \geq 1 .
$$

Remark 2 The requirement of uniform boundedness is satisfied by several functions, such as Legendre polynomials, sinusoidal or Bessel functions (for Bessel functions, we refer to [20]). These functions represent standard choices for the modal basis in a HiMod reduction $[17,22,27]$.

For the sake of brevity, we include the proof of Lemma 2 in the proof of the following result.

Theorem 2 Let $\varphi_{k}$ be the eigenfunction, solution to problem (10) completed with homogeneous Neumann boundary conditions. If $f \in H^{4}(\gamma)$ and satisfies homogeneous Neumann boundary conditions as $\varphi_{k}$, then there exists a constant $C_{3, s}$, independent of $m$, such that, for $s=0,1$,

$$
\left\|\mathcal{R}_{m} f\right\|_{H^{s}(\gamma)} \leq C_{3, s}\left(\frac{1}{m+1}\right)^{\frac{3-s}{d-1}}\|f\|_{H^{4}(\gamma)} .
$$

In addition, if the basis functions $\left\{\varphi_{k}\right\}_{k}$ are uniformly bounded with respect to $k$, then there exists a constant $C_{4, s}$, independent of $m$, such that

$$
\left\|\mathcal{R}_{m} f\right\|_{H^{s}(\gamma)} \leq C_{4, s}\left(\frac{1}{m+1}\right)^{\frac{4-s}{d-1}}\|f\|_{H^{4}(\gamma)} .
$$


Proof If $f \in H^{4}(\gamma)$, it is possible to mimic the procedure adopted in Lemma 1, by working directly on the coefficients of the generalized Fourier series for $\mathcal{L}_{S} f$. However, we can improve the previous estimates by explicitly exploiting the Neumann boundary conditions. Via integration by parts and thanks to (10), we have

$$
\begin{aligned}
{\widehat{\mathcal{L}_{S} f_{k}}=} & -\int_{\gamma} \nabla \cdot(\mu \nabla f) \varphi_{k} d \mathbf{y}+\int_{\gamma} \tilde{\sigma} f \varphi_{k} d \mathbf{y} \\
= & \frac{1}{\lambda_{k}}\left[\int_{\gamma} \nabla \cdot(\mu \nabla f) \nabla \cdot\left(\mu \nabla \varphi_{k}\right) d \mathbf{y}-\int_{\gamma} \tilde{\sigma} \nabla \cdot(\mu \nabla f) \varphi_{k} d \mathbf{y}\right. \\
& \left.-\int_{\gamma} \tilde{\sigma} \nabla \cdot\left(\mu \nabla \varphi_{k}\right) f d \mathbf{y}+\int_{\gamma} \tilde{\sigma}^{2} f \varphi_{k} d \mathbf{y}\right] \\
= & \frac{1}{\lambda_{k}}\left[\int_{\gamma} \nabla \cdot(\mu \nabla(\nabla \cdot(\mu \nabla f))) \varphi_{k} d \mathbf{y}-\int_{\gamma} \tilde{\sigma} \nabla \cdot(\mu \nabla f) \varphi_{k} d \mathbf{y}\right. \\
& -\int_{\gamma} \nabla \cdot(\mu \nabla(\widetilde{\sigma} f)) \varphi_{k} d \mathbf{y}+\int_{\gamma} \tilde{\sigma}^{2} f \varphi_{k} d \mathbf{y} \\
& \left.-\int_{\partial \gamma} \nabla(\nabla \cdot(\mu \nabla f)) \cdot \mathbf{n} \mu \varphi_{k} d s+\int_{\partial \gamma} \nabla(\tilde{\sigma} f) \cdot \mathbf{n} \mu \varphi_{k} d s\right] .
\end{aligned}
$$

Thus, by combining (15) with (24), and by exploiting the trace inequality together with the $L^{2}(\gamma)$-orthonormality of functions $\left\{\varphi_{k}\right\}_{k}$, we obtain the relation

$$
\left|\hat{f}_{k}\right| \leq C \frac{1+\tau^{2}\left\|\varphi_{k}\right\|_{H^{1}(\gamma)}}{\lambda_{k}^{2}}\|f\|_{H^{4}(\gamma)}
$$

with $C$ a constant depending on the problem data and $\tau$ the constant of the trace inequality. In addition, since from the $L^{2}(\gamma)$-orthonormality of the eigenfunctions follows that $\left\|\varphi_{k}\right\|_{H^{1}(\gamma)} \simeq \sqrt{\lambda_{k}}$, we obtain (20). From (25), if $\left\{\varphi_{k}\right\}_{k}$ are uniformly bounded with $k$, then (21) follows.

Estimates (22) and (23) are an immediate consequence of (20) and (21) when we apply the arguments used in Theorem 1.

\section{4 e-HiMod Reduction}

The ultimate goal of the procedure proposed in this paper is to construct an "educated" extension of the generic boundary conditions imposed on the lateral surface of $\Omega$. To this aim, we exploit the theory in the previous section and build a basis $\left\{\varphi_{k}\right\}_{k}$ which efficiently includes condition $\mathcal{B} u=0$ on $\Gamma_{w}$ in an essential way. We refer to such a basis as educated basis and we denote the corresponding hierarchical model reduction by $e$-HiMod approach.

The basic e-HiMod procedure consists of the following steps:

1. split the problem along the axial (one-dimensional) and the transverse direction, respectively;

2. solve the $(d-1)$-dimensional SLE problem (10) associated with $\mathcal{L}_{S}$ on the transverse fiber $\gamma$, to obtain the modal basis $\left\{\varphi_{k}\right\}$;

3. assemble the HiMod block tridiagonal matrix associated with the one-dimensional mainstream coupled problems (6);

4. solve the HiMod system. 
As the choice of $\mathcal{L}_{S}$ is pretty free, a possible approach is to choose it as the symmetric part of the operator to solve. The rationale of this choice is that the e-HiMod basis will be more educated about the problem to solve. This choice-although not strictly necessaryis expected to bring a reduction of the number of modes to attain a given accuracy. In particular, in our ADR problem, the HiMod framework makes sense when the convective part dominates in the axial direction, so that the transverse dynamics is described by an almost purely diffusive operator, like $\mathcal{L}_{S}$. Nevertheless, in the search of a trade-off between accuracy and efficiency, some remarks are in order. (i) For a generic operator $\mathcal{L}$ with space dependent coefficients, solving the SLE on the symmetric part of the operator, may be fairly involved. The burden introduced by the solution of the SLE with space-dependent coefficients and the numerical errors associated makes this approach not optimal. We resort therefore to a constant-coefficient approximation of the symmetric part of $\mathcal{L}$ (by averaging the spacedependent coefficients), whose numerical solution is much easier if not trivial. We consider this as the best trade-off between basis education and overall efficiency. By no means this implies we can solve only problems with constant coefficients, as we demonstrate in Sect. 5. (ii) With a similar perspective, for an unsteady problem with time-dependent coefficients (out of the goal of the present paper), the "most-educated" basis should be recomputed at each time step. However, the computational advantage of the basis customization is burdened by the SLE solves at each time step. In this case, it is worth resorting to a template timeaveraged constant-coefficient symmetric problem to be solved once. (iii) When $\Omega \subset \mathbb{R}^{3}$ (i.e., $\gamma$ is a two-dimensional fiber), problem (10) can be further split into the tensor product of one-dimensional problems, as we will detail in Sect. 4.2.

\subsection{D e-HiMod Reduction in Rectangular Domains}

Now, we exemplify the e-HiMod reduction procedure on a two-dimensional and on a three-dimensional counterpart of problem (1). 2D problems are intended to provide a comprehensive assessment to highlight multiple aspects of the method, while 3D problems point to more practical applications (out of the scope of the paper).

In (1) we prescribe the homogeneous Robin condition $\mu \nabla u \cdot \mathbf{n}+\chi u=0$ on $\Gamma_{w}$, being $\Omega=(0, L) \times(0,1)$ and by selecting constant problem data according to what observed in the previous section. First, we re-write the weak form of the problem by including the Robin condition as: find $u=u(x, y) \in V=H_{\Gamma_{1} \cup \Gamma_{2}}^{1}(\Omega)$ such that

$$
\begin{aligned}
& \int_{0}^{L} \int_{0}^{1} \mu\left(\frac{\partial u}{\partial x}(x, y) \frac{\partial v}{\partial x}(x, y)+\frac{\partial u}{\partial y}(x, y) \frac{\partial v}{\partial y}(x, y)\right) d x d y+\int_{0}^{L} \chi u(x, 1) v(x, 1) d x \\
& +\int_{0}^{L} \chi u(x, 0) v(x, 0) d x+\int_{0}^{L} \int_{0}^{1}\left(\beta_{1} \frac{\partial u}{\partial x}(x, y) v(x, y)+\beta_{2} \frac{\partial u}{\partial y}(x, y) v(x, y)\right) d x d y \\
& +\int_{0}^{L} \int_{0}^{1} \sigma u(x, y) v(x, y) d x d y=\int_{0}^{L} \int_{0}^{1} f(x, y) v(x, y) d x d y \quad \forall v \in V .
\end{aligned}
$$

Now, we solve the SLE problem (10) with $w=1$ to generate the educated modal basis $\left\{\varphi_{k}\right\}_{k=}^{m} 1$ characterizing the HiMod approximation (4). The $L^{2}(\gamma)$-orthonormality of the eigenfunctions $\left\{\varphi_{k}\right\}_{k}$ simplifies the first two HiMod coefficients in (7) to

$$
r_{k, j}^{11}(x)=\mu \delta_{j k}, r_{k, j}^{10}(x)=\beta_{1} \delta_{j k},
$$


respectively. The third HiMod coefficient reduces to

$$
r_{k, j}^{00}(x)=\int_{0}^{1} \beta_{2} \varphi_{j}^{\prime}(y) \varphi_{k}(y) d y+\lambda_{j} \delta_{j k}
$$

since, by exploiting problem (10) and the $L^{2}(\gamma)$-orthonormality of functions $\left\{\varphi_{k}\right\}_{k}$, it holds

$$
\begin{aligned}
& \int_{0}^{1}\left(\mu \varphi_{j}^{\prime}(y) \varphi_{k}^{\prime}(y)+\sigma \varphi_{j}(y) \varphi_{k}(y)\right) d y-\mu\left[\varphi_{j}^{\prime}(y) \varphi_{k}\right]_{0}^{1} \\
& \quad=\int_{0}^{1}\left(\mu \varphi_{j}^{\prime}(y) \varphi_{k}^{\prime}(y)+\sigma \varphi_{j}(y) \varphi_{k}(y)\right) d y+\chi\left[\varphi_{j} \varphi_{k}\right]_{0}^{1}=\int_{0}^{1} \lambda_{j} \varphi_{j}(y) \varphi_{k}(y) d y=\lambda_{j} \delta_{j k} .
\end{aligned}
$$

Exploiting this relation, the assembly cost of the e-HiMod matrix significantly reduces. In addition, in the absence of a vertical convection (i.e., for $\beta_{2}=0$ ), the sparsity pattern becomes block-diagonal. This generally leads to a significant cost reduction for the linear algebra, as we have already noticed in Fig. 3.

Remark 3 Non-homogeneous boundary conditions can be in general solved by appropriate lifting functions. While for complex general cases, the identification of an appropriate lifting is not trivial, for simple problems (yet covering many applications) we may follow simple procedures based on a modal expansion of the data in the domain of interest (see Remark 1 in [27] for further details). In the subsequent sections we present numerical test cases with non-homogeneous conditions solved in this way. Similarly, the case where different types of boundary conditions are prescribed on different portions of $\Gamma_{w}$ can be classically solved by a domain decomposition approach [35].

For more general cases (e.g., with numerical boundary conditions prescribed by the solution of other solvers), the approach may require more specific techniques that are beyond the purpose of the present paper.

Remark 4 The solution to the one-dimensional SLE problem (10) can be obtained, in general, analytically or after the numerical root-finding of a nonlinear function [8]. For instance, for the mixed homogeneous boundary conditions $\varphi_{k}(0)=0$ and $\varphi_{k}^{\prime}(1)+\chi \varphi_{k}(1)=0$, the eigenvalues of problem (10) coincide with the roots of the nonlinear equation $\chi \tan \left(\lambda_{k}\right)+\lambda_{k}=$ 0 , while the eigenfunctions are given by $\varphi_{k}(y)=\sin \left(\lambda_{k} y\right)$, so that we deal with basis functions which are uniformly bounded with $k$. When possible, the analytical approach reduces all the numerical errors that may show up in solving the differential problem at hand (we also refer to Remark 7 for some additional considerations).

\subsection{D e-HiMod Reduction in Slab Domains}

We hierarchically reduce now problem (1) in the parallelepiped domain $\Omega=\left(0, L_{x}\right) \times$ $\left(0, L_{y}\right) \times\left(0, L_{z}\right)$ of Fig. 1 , by assigning the homogeneous Robin data $\mu \nabla u \cdot \mathbf{n}+\chi u=0$ on $\Gamma_{w}$. The problem data are again assumed constant. For this purpose, we generalize the procedure in the previous section to a 3D setting. In particular, to identify the educated modal basis associated with the selected boundary conditions, we solve the SLE problem (10) on the transverse fiber $\gamma$ by taking advantage of the Cartesian structure of $\Omega$. In this way problem (10) can be turned into a pair of one-dimensional SLE problems, whose solutions can be 
computed analytically. For complex geometries tensor product may be not an option. In this case, other solutions are demanded [17].

For simplicity, we set $\widetilde{\sigma}=0$ in (8) since the reactive term just shifts the spectrum of the operator $\mathcal{L}_{S}$. Then, we factorize the eigenfunction in (10) as

$$
\varphi_{k}(y, z)=\varphi_{y, p(k)}(y) \varphi_{z, q(k)}(z),
$$

where $p(k)$ and $q(k)$ are indices related to the $y$ - and to the $z$-coordinate, respectively used to identify the $k$-th 2D modal function $\varphi_{k}$. Factorization (26) leads to the one-dimensional eigenvalue problems

$$
\begin{aligned}
& \left\{\begin{array}{l}
-\mu \varphi_{y, p(k)}^{\prime \prime}(y)=\lambda_{y, p(k)} \varphi_{y, p(k)}(y) \quad \text { in }\left(0, L_{y}\right) \\
\mu \varphi_{y, p(k)}^{\prime}(0)+\chi \varphi_{y, p(k)}(0)=0 \\
\mu \varphi_{y, p(k)}^{\prime}\left(L_{y}\right)+\chi \varphi_{y, p(k)}\left(L_{y}\right)=0
\end{array}\right. \\
& \left\{\begin{array}{l}
-\mu \varphi_{z, q(k)}^{\prime \prime}(z)=\lambda_{z, q(k)} \varphi_{z, q(k)}(z) \quad \text { in }\left(0, L_{z}\right) \\
\mu \varphi_{z, q(k)}^{\prime}(0)+\chi \varphi_{z, q(k)}(0)=0 \\
\mu \varphi_{z, q(k)}^{\prime}\left(L_{z}\right)+\chi \varphi_{z, q(k)}\left(L_{z}\right)=0,
\end{array}\right.
\end{aligned}
$$

with $\lambda_{y, p(k)}$ and $\lambda_{z, q(k)}$ the eigenvalue associated with the eigenfunction $\varphi_{y, p(k)}$ and $\varphi_{z, q(k)}$, respectively. Thus, the eigenpair $\left(\varphi_{k}(y, z), \lambda_{k}\right)$ solution to the $2 \mathrm{D}$ problem (10) on the slice $\gamma$ reads

$$
\left(\varphi_{k}(y, z), \lambda_{k}\right)=\left(\varphi_{y, p(k)}(y) \varphi_{z, q(k)}(z), \lambda_{y, p(k)}+\lambda_{z, q(k)}\right) .
$$

Consistently with the analysis of the previous section, the eigenvalues $\lambda_{k}$ have to be sorted into a non-decreasing sequence. We select indices $p(k)$ and $q(k)$ to identify the eigenvalue $\lambda_{k}$ in (29) via the following algorithm hereafter denoted by ESA (Eigenvalue Sorting Algorithm).

Let $m$ be given. Then, we perform the following steps.

(i) For $k=1$, we set $\lambda_{1}=\lambda_{y, 1}+\lambda_{z, 1}$, i.e., $p(1)=1$ and $q(1)=1$ identify $k=1$.

(ii) While $k \leq m$, do

- compute $\lambda_{y, p(k)+1}+\lambda_{z, q(k)}$ and $\lambda_{y, p(k)}+\lambda_{z, q(k)+1}$ and store these values in the list of the eigenvalues to examine;

- compute the minimal element in the list of the eigenvalues to examine; this will be assigned to $\lambda_{k+1}$; correspondingly, we assign $p(k+1)$ and $q(k+1)$;

- increment $k$.

A schematization of ESA is depicted in Fig. 4. In particular, the diagram refers for simplicity to (27), for $L_{y}=\pi$ and $L_{z}=3 \pi / 2$, completed with homogeneous Dirichlet boundary conditions, i.e., to a test case where the eigenvalues can be calculated exactly. The light-grey boxes refer to eigenvalues already examined, while the white boxes identify the eigenvalues that have to be still checked. In the boxes we report three numbers with the following notation $\left(\lambda_{y, p(k)}, \lambda_{z, q(k)}\right) \lambda_{k}$, while the numbers in brackets, outside the boxes, denote the index $k$.

When dealing with a 2D SLE problem, eigenvalues may have a multiplicity strictly greater than one. In the proposed algorithm, when this repetition occurs, either one of the repeated nodes or the other (see the dark-grey boxes in Fig. 4) is removed from the list. For instance, in Fig. 4, after the detection of the simple eigenvalue $\lambda_{4}=5$, we obtain the next candidate, i.e., the value 5.77 , twice. Thus, one of the two occurrences is eliminated from the tree.

Remark 5 When fiber $\gamma$ exhibits a dominant dimension, for instance, $L_{y} \gg L_{z}$, it is worth using a different number of modal basis functions along the direction $y$ and $z$, respectively. 


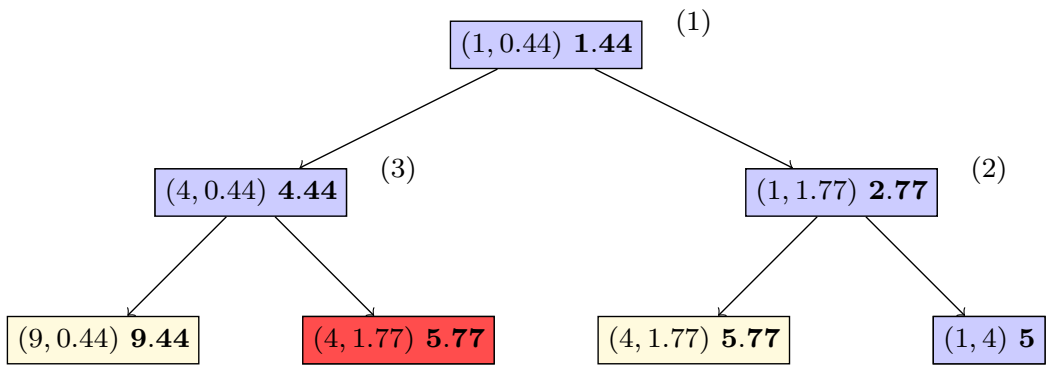

Fig. 4 Schematization of ESA to select the 2D eigenvalues $\lambda_{k}$ in (29)

In this case, the eigenvalues associated with $z$-direction are larger than the ones related to $y$-direction, as indicated by formula (12). Hence, since the truncation error scales with the inverse of the first truncated eigenvalue, less modal functions need to be employed along $z$-direction than $y$-direction. Thus, if the ratio $L_{z} / L_{y}$ is significantly small, out of $m$ modes we will select $m-1$ modes in the $y$-direction and a single mode along $z$, so that the tree of Fig. 4 becomes extremely unbalanced, following only the $y$-side of the branch.

\subsection{Error Analysis}

To analyze the error characterizing the e-HiMod reduction of problem (1), we first compute the error associated with the modal discretization (semi-discrete problem), and then we include the error due to the finite element approximation of the axial dynamics.

As for the semi-discretization error, we have the following result.

Theorem 3 Let $u \in H^{2}(\Omega)$ be the weak solution to the full problem (1), with $\Omega \subset \mathbb{R}^{d}$, and let $P_{m} u$ denote the orthogonal projection of $u$ onto the HiMod space $V_{m}$ in (3), being $\left\{\varphi_{k}\right\}_{k=1}^{m}$ an educated modal basis. Then, there exists a constant $\widetilde{C}_{1, s}$, independent of $m$, such that, for $s=0,1$,

$$
\left\|u-P_{m} u\right\|_{H^{s}(\Omega)} \leq \widetilde{C}_{1, s}\left(\frac{1}{m+1}\right)^{\frac{2-s}{d-1}}\|u\|_{H^{2}(\Omega)} .
$$

Moreover, if $u \in H^{2 p}(\Omega)$, with $p \geq 2$, and $\mathcal{L}_{\widetilde{\mathcal{L}}}^{r} u$ satisfies compatible boundary conditions up to order $p-1$, then there exists a constant $\widetilde{C}_{2, s}$, independent of $m$, such that, for $s=0,1$,

$$
\left\|u-P_{m} u\right\|_{H^{s}(\Omega)} \leq \widetilde{C}_{2, s}\left(\frac{1}{m+1}\right)^{\frac{2 p-s}{d-1}}\|u\|_{H^{2 p}(\Omega)} .
$$

Proof By exploiting the density of the space $V_{\infty}$ in $V$ for the modal representation of $u$, we have

$$
\begin{aligned}
& \left\|u-P_{m} u\right\|_{L^{2}(\Omega)}^{2}=\int_{\Omega_{1 D}} \int_{\gamma}\left[\sum_{k=m+1}^{+\infty} u_{k}(x) \varphi_{k}(\mathbf{y})\right]^{2} d \mathbf{y} d x \\
& =\int_{\Omega_{1 D}}\left\|\left(u-P_{m} u\right)(x)\right\|_{L^{2}(\gamma)}^{2} d x .
\end{aligned}
$$

Estimates (30) and (31) for $s=0$ now follow from Theorem 1, after identifying $\mathcal{R}_{m} f$ with $\left(u-P_{m} u\right)(x)$. Similar arguments can be used for the error estimates with respect to the $H^{1}(\Omega)$-norm. 
Now, we consider the fully discretized solution $u_{m}^{h}$, obtained by completing the modal expansion with an approximation of the axial dynamics via finite elements. So far we have assumed to employ a piecewise linear discretization along $\Omega_{1 D}$. The next results are more general and refer to finite elements of generic order $r$.

Theorem 4 Let $u \in H^{s}(\Omega)$, with $s \geq 2$, be the weak solution to the full problem (1), with $\Omega \subset \mathbb{R}^{d}$. Then, the error associated with the e-HiMod reduction satisfies the a priori estimate

$$
\left\|u-u_{m}^{h}\right\|_{H^{1}(\Omega)} \leq C\left(h^{q}+m^{-l}\right)\|u\|_{H^{s}(\Omega)},
$$

with $q=\min (s-1, r)$ and $l=1 /(d-1)$. In particular, if $u \in H^{2 p}(\Omega)$, with $p \geq 2$, and $\mathcal{L}_{S}^{r} u$ satisfies compatible boundary conditions up to order $p-1$, then $l=(2 p-1) /(d-1)$.

Proof Via Céa's Lemma and the triangle inequality, it follows

$$
\begin{aligned}
\left\|u-u_{m}^{h}\right\|_{H^{1}(\Omega)} & \leq C \inf _{v_{m}^{h} \in V_{m}^{h}}\left\|u-v_{m}^{h}\right\|_{H^{1}(\Omega)} \\
& \leq C\left(\left\|u-v_{m}\right\|_{H^{1}(\Omega)}+\left\|v_{m}-v_{m}^{h}\right\|_{H^{1}(\Omega)}\right),
\end{aligned}
$$

where $v_{m} \in V_{m}$ is the semi-discrete counterpart of the generic e-HiMod function $v_{m}^{h}$. Now, we identify $v_{m}$ with $P_{m} u$. As a consequence, the thesis promptly follows from classical piecewise polynomial approximation results [13] and from Theorem 3.

With similar arguments from Theorem 2 for the specific case of Neumann data, we have the following result (we do not report the proof for brevity):

Theorem 5 Let $u \in H^{4}(\Omega)$ be the weak solution to the full problem (1) completed with homogeneous Neumann conditions on $\Gamma_{w}$ and homogeneous Dirichlet data on $\Gamma_{1} \cup \Gamma_{2}$, with $\Omega \subset \mathbb{R}^{d}$. Let $P_{m} u$ be defined as in Theorem 3 . Then, there exists a constant $\widetilde{C}_{3, s}$ independent of $m$, such that, for $s=0,1$,

$$
\left\|u-P_{m} u\right\|_{H^{s}(\Omega)} \leq \widetilde{C}_{3, s}\left(\frac{1}{m+1}\right)^{\frac{3-s}{d-1}}\|u\|_{H^{4}(\Omega)} .
$$

In addition, if the modal functions $\left\{\varphi_{k}\right\}_{k}$ are uniformly bounded with respect to $k$, then there exists a constant $\widetilde{C}_{4, s}$, independent of $m$, such that

$$
\left\|u-P_{m} u\right\|_{H^{s}(\Omega)} \leq \widetilde{C}_{4, s}\left(\frac{1}{m+1}\right)^{\frac{4-s}{d-1}}\|u\|_{H^{4}(\Omega)} .
$$

Thus, if $u \in H^{s}(\Omega)$, with $s \geq 2$, the error associated with the e-HiMod reduction procedure satisfies the a priori estimate

$$
\left\|u-u_{m}^{h}\right\|_{H^{1}(\Omega)} \leq C\left(h^{q}+m^{-l}\right)\|u\|_{H^{s}(\Omega)},
$$

with $q=\min (s-1, r)$ and $l=(3-s) /(d-1)($ or $l=(4-s) /(d-1)$ for uniformly bounded modal basis functions).

Remark 6 Under suitable assumptions on the boundary $\partial \Omega$ of $\Omega$ and on the boundary data, estimates (32) and (33) can be generalized to control the $L^{2}(\Omega)$-norm of the global error $u-u_{m}^{h}$, with the expected orders $q+1$ and $l+1$. 
Remark 7 When numerically computing the educated modal basis, errors may induce a loose of orthogonality. This reflects into an additional error component. In practice, the numerical modal approximation $\tilde{u}_{m}$ of $u_{m}$ may not coincide with the orthogonal projection $P_{m} u$. In fact, the coefficients of the expansion of $\tilde{u}_{m}$ on the selected basis are affected by an error due to the terms $\left(\varphi_{i}, \varphi_{j}\right)$ for $i \neq j$. In this case, denoting by $\varepsilon$ the orthogonality error $\left(\varepsilon \leq \max _{i j}\left|\left(\varphi_{i}, \varphi_{j}\right)\right|\right)$, we argue that the error $u-u_{m}^{h}$ features an additional component proportional to $\varepsilon \lambda_{1}^{-1}$. In our numerical results the impact of this error never prevented the convergence of the scheme, as we verified that $\varepsilon$ is significantly smaller than the discretization error.

Remark 8 Integration cannot be performed analytically, since the modal functions are in general not polynomials. In our numerical tests, we resort to a 64 node Gauss-Legendre formula along both the $y$ and the $z$ directions. The accuracy of this formula is enough to qualify integration error as of higher order with respect to the expected e-HiMod convergence rate. Also, as the assembly cost is affected by the large number of quadrature nodes, in our test cases we verified that using a cheaper 32 node formula is enough to manage a number of modes up to 20, with no evident impact on the accuracy.

\section{Numerical Assessment of the e-HiMod Procedure}

We consider both 2D and 3D test cases. The two-dimensional e-HiMod code is developed in Mat lab, while the 3D tests are implemented in LifeV (https://cmcsforge.epfl.ch/doxygen/ lifev/). In Matlab, systems were small enough to be solved with the built-in solvers. While this is not necessarily possible with other environments, the reduction of the number of dof may, in general, enable using solvers otherwise out of reach. In 3D, LifeV resorts to standard Trilinos solvers (GMRes). As we have anticipated, devising specific multilevel solvers exploiting the hierarchical structure of the method-partially explored in [5] —is one of the follow-ups of the present work. Numerical results are validated against analytical solutions when available, and reference finite element approximations computed via FreeFem ++ [18] on fine enough meshes otherwise. Some tests provide a specific quantitative performance analysis, in others we limit to a qualitative check for the sake of brevity.

\subsection{Verification}

We start by investigating the consistency of the e-HiMod procedure with a reference finite element solution computed on a fine mesh in absence of an analytical solution. In particular, we focus on a 2D setting and we consider different choices for the boundary data.

Let us consider the problem

$$
\begin{cases}-\Delta u+\boldsymbol{\beta} \cdot \nabla u=f & \text { in } \Omega=(0,6) \times(0,1) \\ u=g_{D} \text { on } \Gamma_{\text {in }}, & \nabla u \cdot \mathbf{n}+\chi u=g_{R} \text { on } \Gamma_{\text {out }} \\ \rho_{1} \nabla u \cdot \mathbf{n}+\rho_{2} u=0 & \text { on } \Gamma_{u p} \cup \Gamma_{\text {down }},\end{cases}
$$

where $\boldsymbol{\beta}=(20,0)^{T}$ represents a horizontal convective field, $\Gamma_{\text {in }}=\{0\} \times[0,1], \Gamma_{\text {out }}=$ $\{6\} \times[0,1], g_{D}$ and $g_{R}$ are given functions, $\chi$ is a constant, and the forcing term $f$ models two elliptical sources localized in the left-portion of the domain, being $f(x, y)=\chi_{E_{1} \cup E_{2}}(x, y)$, with $E_{1}=\left\{(x, y) \in \Omega:(x-1.5)^{2}+0.4(y-0.25)^{2} \leq 0.01\right\}$ and $E_{2}=\{(x, y) \in$ $\left.\Omega:(x-1.5)^{2}+0.4(y-0.75)^{2} \leq 0.01\right\}$ (see Fig. 5). Condition on $\Gamma_{w}=\Gamma_{u p} \cup \Gamma_{\text {down }}$ 
Fig. 5 Schematization of the 2D test case setting for the e-HiMod verification

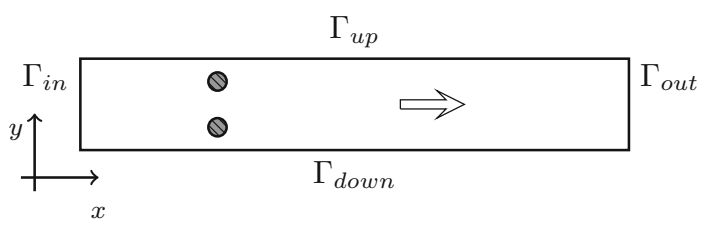

prescribes Robin, Dirichlet or Neumann data according to the parameters $\rho_{1}, \rho_{2}$, which can take different values on different portions of the lateral boundary, being $\Gamma_{u p}=[0,6] \times\{1\}$ and $\Gamma_{\text {down }}=[0,6] \times\{0\}$.

We test two combinations of boundary conditions on $\Gamma_{u p}$ and $\Gamma_{\text {down }}$, i.e., Dirichlet/Robin and Robin/Robin data, respectively. At the intersection of the different portions of the boundary, data are prescribed to be compatible. As reference solution, we take the continuous piecewise linear approximation computed on a structured uniform grid with mesh sizes $h_{x}=h_{y}=0.0025$. For the e-HiMod approximation, linear finite elements discretize $\Omega_{1 D}$, on $\mathcal{T}_{h}$ uniform partition of step $h=0.01$, while varying the number of educated modes along $\gamma$.

Dirichlet/Robin Data We assign the Robin condition $\nabla u \cdot \mathbf{n}=-3(u-0.06)$ on $\Gamma_{\text {down }}$, and the Dirichlet data $u=0.05$ on $\Gamma_{u p}$. In Fig. 6, top we show the contour plot of the reference finite element approximation. The Robin data on $\Gamma_{\text {down }}$ warps downward the horizontal dynamics induced by the convective field, so the effect of the two forcing terms on the solution is different and clearly detectable.

We compute the e-HiMod approximation by gradually increasing the modal index $m$. Here, e-Himod $(m)$ denotes the solution associated with $m$ educated modal functions given by the first $m$ eigenfunctions. Figure 6 shows the contour plot of the e-HiMod$(m)$ approximation, for $m=2,4,8$. As expected, the quality of the reduced solution improves when $m$ increases. For $m=8$, the solution fully overlaps to the reference one.

Robin/Robin Data We now assign non-homogeneous Robin conditions on both $\Gamma_{u p}$ and $\Gamma_{\text {down }}$, namely we impose $\nabla u \cdot \mathbf{n}=-3(u-0.06)$ on $\Gamma_{u p}$ and $\nabla u \cdot \mathbf{n}=-3(u-0.05)$ on $\Gamma_{\text {down }}$, respectively.

In Fig. 7 we compare the e-HiMod $(m)$ approximation corresponding to $m=2,4,8$ (second-fourth row) with the reference solution (first row), confirming the trend of the previous case.

Space-Dependent Coefficients In Fig. 8 we test the e-HiMod procedure with non-constant coefficient problems. In this case, we add the reactive term $\sigma u$ in (34), where the coefficient $\sigma$ coincides with the characteristic function associated with two circular subsets of the domain. The same Robin data is assigned on $\Gamma_{u p}$ and $\Gamma_{\text {down }}$, while the source term $f$ is set equal to zero. Also in this case, the convergence of the e-HiMod solution to the reference one is evident when $m$ increases.

\subsection{Effectiveness}

We quantify the effectiveness of the e-HiMod reduction procedure with respect to a standard $2 \mathrm{D}$ finite element approximation in terms of number of degrees of freedom (dof). We consider problem (34) by assigning the non-homogeneous Robin conditions $\nabla u \cdot \mathbf{n}=-3(u-0.06)$ on $\Gamma_{u p}$ and $\nabla u \cdot \mathbf{n}=-3(u-0.05)$ on $\Gamma_{d o w n}$, respectively while selecting $f=10 \chi_{F_{1} \cup F_{2}}(x, y)$, 


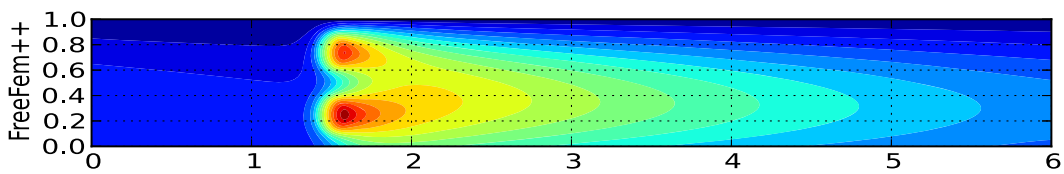

0.13

0.11

0.09

0.07

0.05

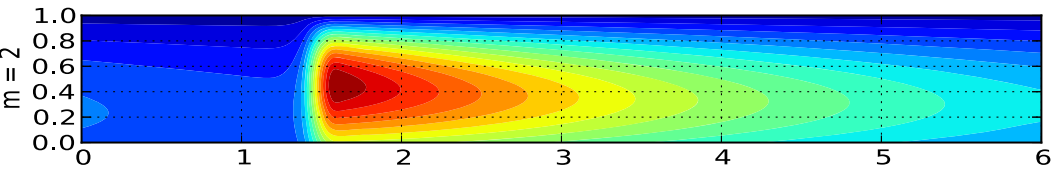

0.13

0.11

0.09

0.07

0.05

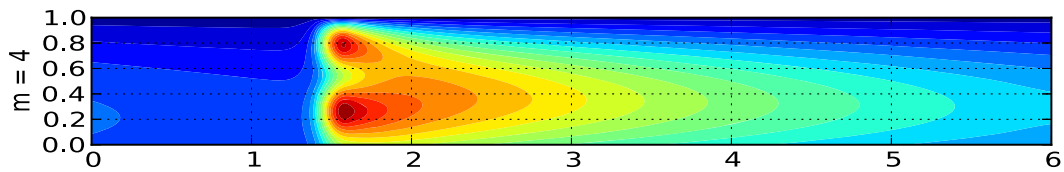

0.13

0.11

0.09

0.07

0.05

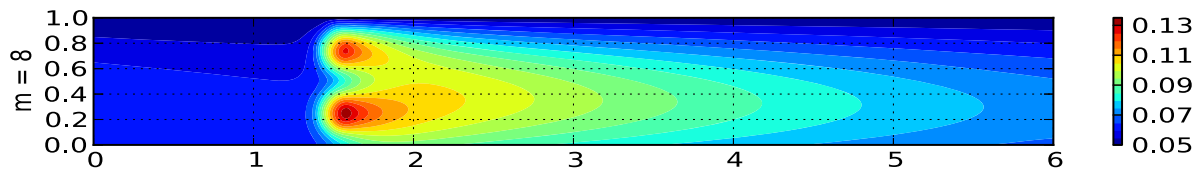

Fig. 6 Dirichlet/Robin data: reference finite element solution (top); e-HiMod( $m$ ) reduced solution for $m=2$, 4,8 (second-fourth row)
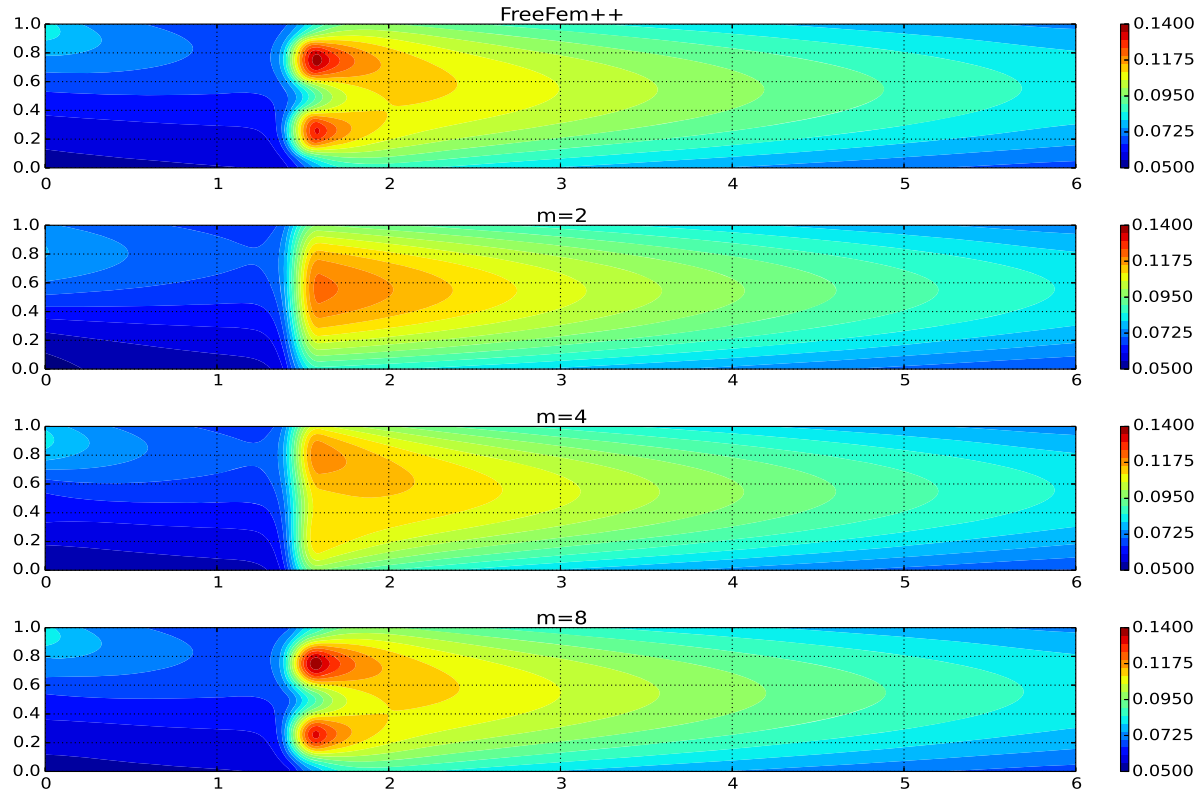

Fig. 7 Robin/Robin data: reference finite element solution (top); e-HiMod( $m$ ) reduced solution for $m=2$, 4, 8 (second-fourth row)

with $F_{1}=\left\{(x, y) \in \Omega:(x-3)^{2}+0.4(y-0.25)^{2} \leq 0.01\right\}$ and $F_{2}=\{(x, y) \in \Omega$ : $\left.(x-1.5)^{2}+0.4(y-0.75)^{2} \leq 0.01\right\}$. Notice that the two sources are not aligned with any of the Cartesian axes. 

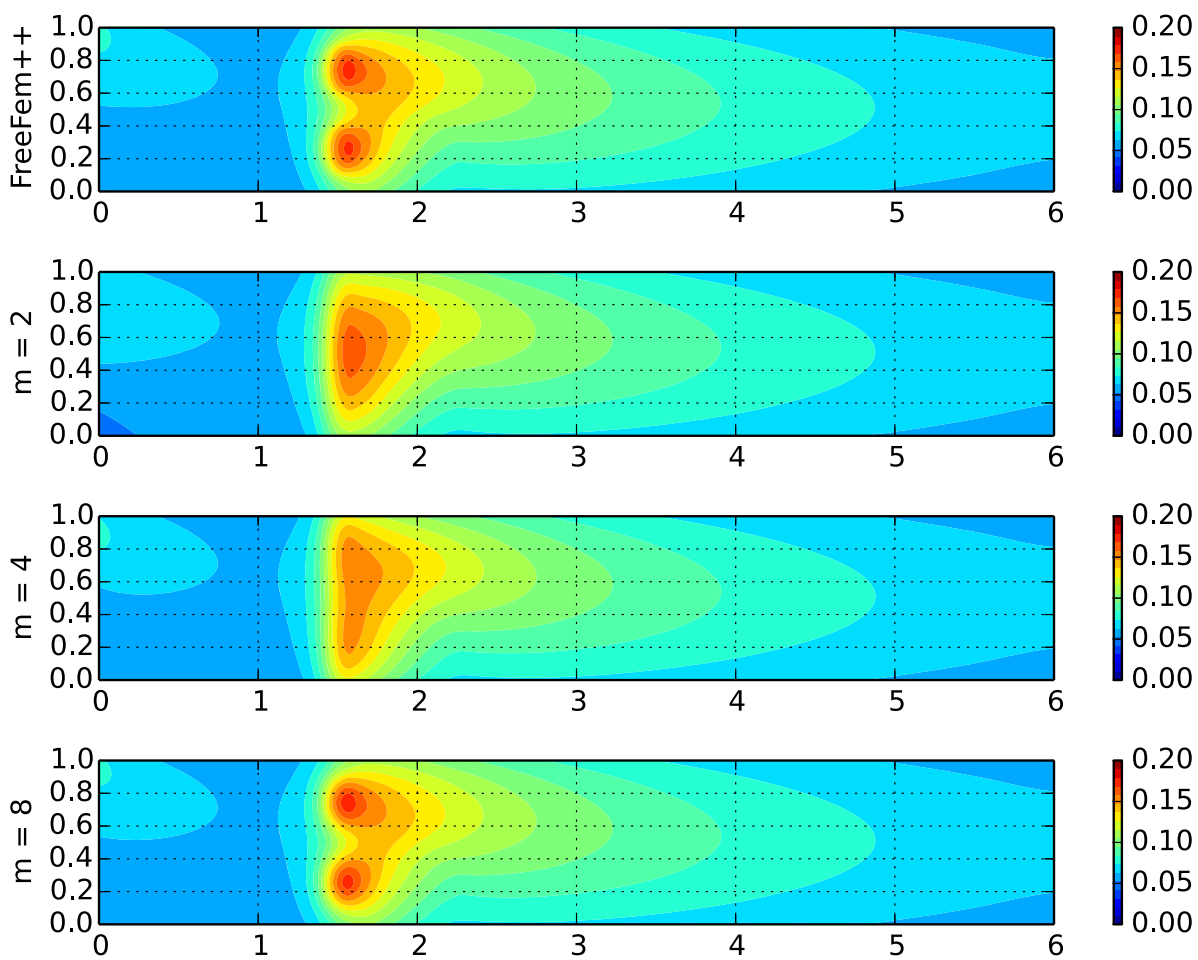

Fig. 8 Space-dependent coefficients: reference finite element solution (top); e-HiMod( $m$ ) reduced solution for $m=2,4,8$ (second-fourth row)

When the leading dynamics is aligned with the supporting fiber $\Omega_{1 D}$, the e-HiMod approach effectively reduces the number of dof, without giving up accuracy. To show this, we compute: a high-resolution linear finite element approximation based on a uniform structured mesh of sizes $h_{x}=0.01, h_{y}=0.01$ (Fig. 9, first row); a low-resolution linear finite element approximation obtained by increasing $h_{y}$ to 0.2 (Fig. 9, second row); the e-HiMod $(m)$ approximation associated with $m=1$ (Fig. 9, fourth row) and $m=5$ (Fig. 9, fifth row), with $\mathcal{T}_{h}$ a uniform partition of $\Omega_{1 D}$ of step size $h=0.01$ in both the cases. Consequently, the number of dof of the four approximations is 60,000 (high-resolution reference solution), 3000 (low-resolution reference solution), 600 (e-HiMod(1)) and 3000 (e-HiMod(5)), respectively.

The e-HiMod(5) approximation perfectly matches the high-resolution reference approximation, albeit obtained by solving a linear system whose dimension is 20 times smaller compared with the finite element one. Conversely, the low-resolution finite element approximation demands exactly the same number of dof as the e-HiMod(5) solution but with an evident lower accuracy.

Finally, as expected, the e-HiMod(1) model is too coarse due to the limited transverse information carried by a single mode. Nevertheless, we point out how the e-HiMod(1) solution is slightly more informative than the transverse-averaged finite element solution in Fig. 9, third row, obtained by averaging the 2D ADR problem along the transverse direction. In particular, we compute the averaged solution by preserving the partition along $\Omega_{1 D}$ of size $h=0.01$, still resulting in 600 dof. This confirms the results in [2], where the HiMod approach is compared with the Geometrical Multiscale Approach [33], coupling dimensionally hetero- 


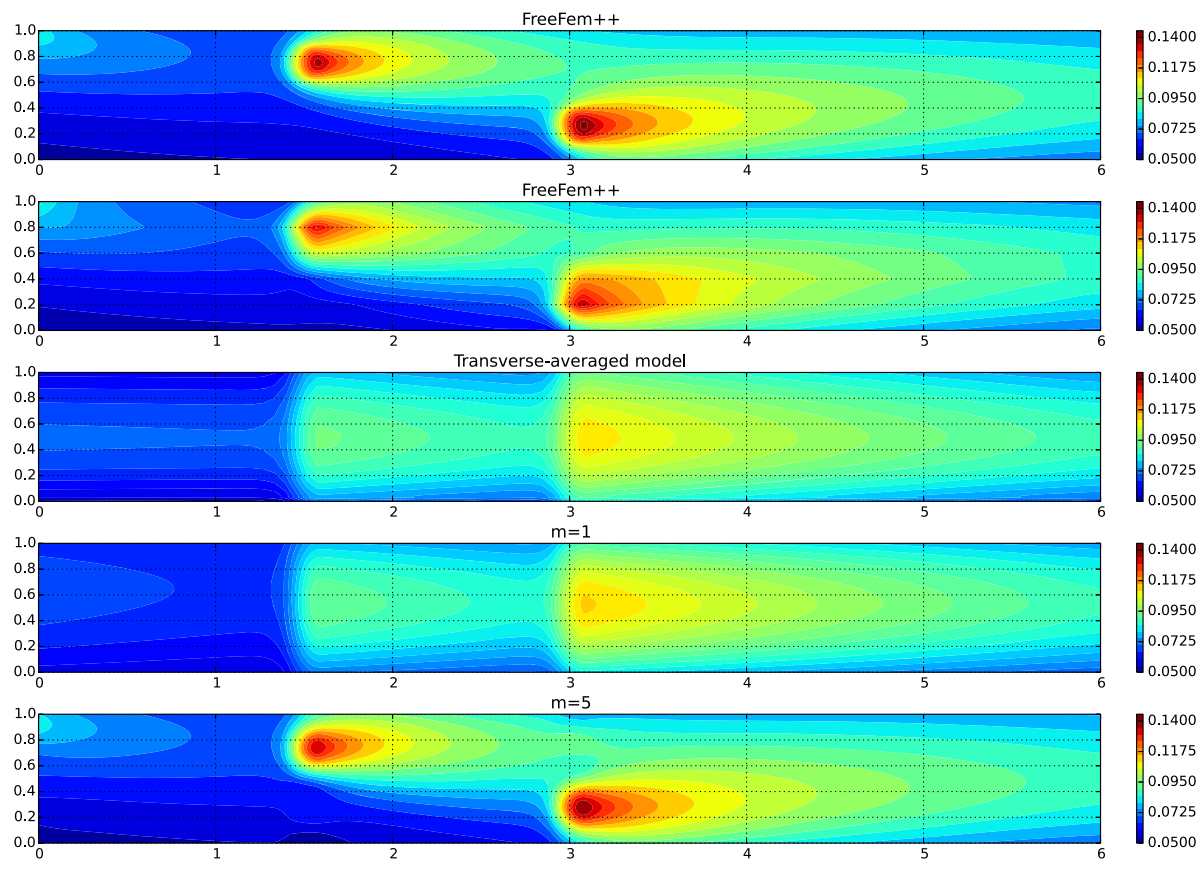

Fig. 9 Robin/Robin data: comparison among 2D linear finite element discretizations on a fine (first) and coarse (second row), the transverse-averaged ADR model (third row), and the e-HiMod(1) and e-HiMod(5) approximations (fourth and fifth row)

geneous problems to cover large portions of a pipe network. The e-HiMod approach provides surrogate approximations (like purely one-dimensional models) yet amenable to be easily refined locally, with computational costs that outperform traditional approaches.

\subsection{Educated Versus Non-educated Modal Bases}

This section highlights the added value provided by an educated modal basis with respect to a standard Fourier basis. For the sake of simplicity, this check is performed in a 2D framework. To this aim, we consider the ADR problem

$$
\begin{cases}-\Delta u+\boldsymbol{\beta} \cdot \nabla u+\sigma u=f & \text { in } \Omega=(0,1)^{2} \\ u=u_{e x} \quad \text { on } \Gamma_{\text {in }} & \nabla u \cdot \mathbf{n}=\nabla u_{\text {ex }} \cdot \mathbf{n} \quad \text { on } \Gamma_{\text {out }} \\ \nabla u \cdot \mathbf{n}+3 u=0 & \text { on } \Gamma_{u p} \cup \Gamma_{\text {down }},\end{cases}
$$

with $\boldsymbol{\beta}=(20,0)^{T}, \sigma=2, \Gamma_{\text {in }}=\{0\} \times[0,1], \Gamma_{\text {out }}=\{1\} \times[0,1], \Gamma_{\text {down }}=[0,1] \times$ $\{0\}, \Gamma_{u p}=[0,1] \times\{1\}$. The source term is selected so that the analytical solution is

$$
\begin{aligned}
u_{e x}= & x y+x+y+\exp (2 x y-y)-1 \\
& -y^{2}[2 x+\exp (2 x-1)+0.1((4 x-6) \exp (2 x-1)-6 x+2)] .
\end{aligned}
$$

We may resort to the Fourier basis $\mathcal{F}_{m}=\{1, \cos (j \pi y), \sin (j \pi y)\}_{j=1}^{m}$ and to a natural treatment of the boundary conditions. This is expected to introduce some error as opposed to the essential treatment of the same conditions with an educated basis. 

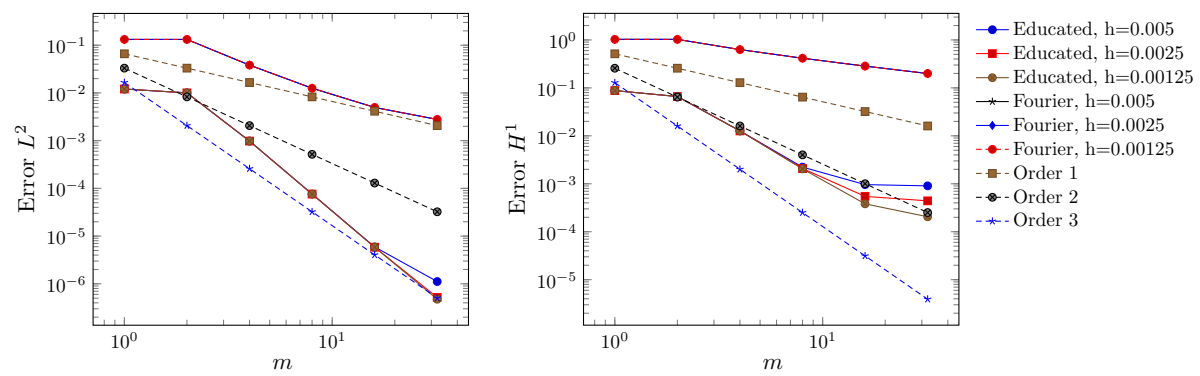

Fig. 10 Educated versus non-educated modal bases: convergence rate of the global error with respect to the $L^{2}(\Omega)$-norm (left) and $H^{1}(\Omega)$-norm (right), for different discretization steps. The reader is referred to the electronic on-line version with colors for a better reading

We compare the convergence of the two HiMod approximations. In Fig. 10 we show the convergence rate of the global error with respect to the $L^{2}(\Omega)$ - (left) and the $H^{1}(\Omega)$-norm (right), for both the educated and the non-educated modal bases. Convergence is attained by both the approaches, even though the HiMod reduction based on $\mathcal{F}_{m}$ is definitely slower compared with the e-HiMod approach. In particular, the Fourier basis leads to a linear convergence for the $L^{2}(\Omega)$-norm, and to a sub-linear rate with respect to the $H^{1}(\Omega)$-norm. Moreover, no sensitivity with respect to $h$ can be appreciated, suggesting that the modal error dominates the one due to the finite element discretization. On the other hand, Theorem 4 predicts a quadratic and a linear convergence rate for the global error of the e-HiMod approximation with respect to the $L^{2}(\Omega)$ - and the $H^{1}(\Omega)$-norm, respectively. Actually, results in Fig. 10 slightly outperform the expected convergence rate in the range of the selected modes.

\subsection{Convergence Analysis}

We perform more quantitative investigations, by assessing the convergence rate of the eHiMod approximation. To this aim, we select a step $h$ small enough to emphasize the modal error, or, alternatively, a large number $m$ of modes to highlight the finite element approximation error. We consider both $2 \mathrm{D}$ and $3 \mathrm{D}$ cases, where the $2 \mathrm{D}$ are intended to provide a clearer evidence of different properties of the method. All the convergence graphs provided hereafter are log-log plots.

\subsubsection{D Analysis}

We consider different choices for the boundary data to be assigned on $\Gamma_{w}$.

Dirichlet/Robin Data The first case test solves the ADR problem

$$
\begin{cases}-\Delta u+\boldsymbol{\beta} \cdot \nabla u+\sigma u=f & \text { in } \Omega=(0,1)^{2} \\ u=u_{\text {ex }} \quad \text { on } \Gamma_{\text {in }}, & u=0 \quad \text { on } \Gamma_{\text {up }} \\ \nabla u \cdot \mathbf{n}+u=3 \quad \text { on } \Gamma_{\text {down }}, & \nabla u \cdot \mathbf{n}=0 \quad \text { on } \Gamma_{\text {out }},\end{cases}
$$

with $\boldsymbol{\beta}=(20,0)^{T}, \sigma=2, \Gamma_{\text {in }}=\{0\} \times[0,1], \Gamma_{\text {out }}=\{1\} \times[0,1], \Gamma_{\text {down }}=[0,1] \times$ $\{0\}, \Gamma_{u p}=[0,1] \times\{1\}$, and $f$ such that the analytical solution is $u_{e x}=4 y^{2}(1-y)(0.75+$ $\left.8 x^{2} y+8 x y^{2}\right)(x-1)^{2}+(1-y)^{2}$. 

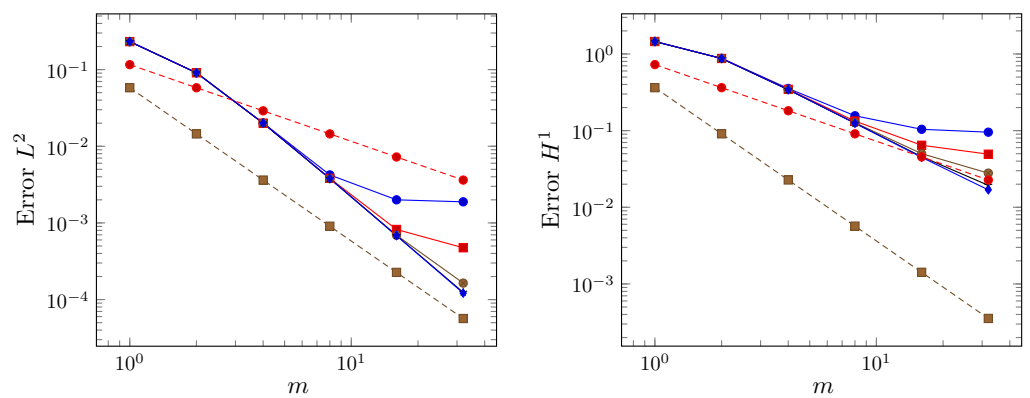

- $\mathrm{h}=0.1$

$-\mathrm{h}=0.05$

$\rightarrow \mathrm{h}=0.025$

* $\mathrm{h}=0.0125$

$\rightarrow \mathrm{h}=0.00625$

- - Order 1

- - Order 2

Fig. $112 \mathrm{D}$ convergence analysis, Dirichlet/Robin data: global error with respect to the $L^{2}(\Omega)$-norm (left) and $H^{1}(\Omega)$-norm (right), for different discretization steps

Table $12 \mathrm{D}$ convergence analysis, Dirichlet/Robin data: global error with respect to the $L^{2}(\Omega)$ (top) and $H^{1}$ norms (bottom)

\begin{tabular}{clllll}
\hline$m$ & $h=0.1$ & $h=0.05$ & $h=0.025$ & $h=0.0125$ & $h=0.00625$ \\
\hline$L^{2}$ norm & & & & \\
1 & $2.32 \mathrm{e}-01$ & $2.32 \mathrm{e}-01$ & $2.32 \mathrm{e}-01$ & $2.32 \mathrm{e}-01$ & $2.32 \mathrm{e}-01$ \\
2 & $9.09 \mathrm{e}-02$ & $9.08 \mathrm{e}-02$ & $9.08 \mathrm{e}-02$ & $9.08 \mathrm{e}-02$ & $9.08 \mathrm{e}-02$ \\
4 & $2.01 \mathrm{e}-02$ & $2.00 \mathrm{e}-02$ & $2.00 \mathrm{e}-02$ & $2.00 \mathrm{e}-02$ & $2.00 \mathrm{e}-02$ \\
8 & $4.23 \mathrm{e}-03$ & $3.82 \mathrm{e}-03$ & $3.79 \mathrm{e}-03$ & $3.79 \mathrm{e}-03$ & $3.79 \mathrm{e}-03$ \\
16 & $2.00 \mathrm{e}-03$ & $8.22 \mathrm{e}-04$ & $6.91 \mathrm{e}-04$ & $6.83 \mathrm{e}-04$ & $6.82 \mathrm{e}-04$ \\
32 & $1.88 \mathrm{e}-03$ & $4.74 \mathrm{e}-04$ & $1.64 \mathrm{e}-04$ & $1.24 \mathrm{e}-04$ & $1.21 \mathrm{e}-04$ \\
$H^{1}$ norm & & & & \\
1 & $1.45 \mathrm{e}+00$ & $1.45 \mathrm{e}+00$ & $1.45 \mathrm{e}+00$ & $1.45 \mathrm{e}+00$ & $1.45 \mathrm{e}+00$ \\
2 & $8.77 \mathrm{e}-01$ & $8.74 \mathrm{e}-01$ & $8.73 \mathrm{e}-01$ & $8.73 \mathrm{e}-01$ & $8.73 \mathrm{e}-01$ \\
4 & $3.55 \mathrm{e}-01$ & $3.45 \mathrm{e}-01$ & $3.43 \mathrm{e}-01$ & $3.42 \mathrm{e}-01$ & $3.42 \mathrm{e}-01$ \\
8 & $1.56 \mathrm{e}-01$ & $1.33 \mathrm{e}-01$ & $1.27 \mathrm{e}-01$ & $1.25 \mathrm{e}-01$ & $1.25 \mathrm{e}-01$ \\
16 & $1.04 \mathrm{e}-01$ & $6.45 \mathrm{e}-02$ & $5.01 \mathrm{e}-02$ & $4.58 \mathrm{e}-02$ & $4.49 \mathrm{e}-02$ \\
32 & $9.54 \mathrm{e}-02$ & $4.93 \mathrm{e}-02$ & $2.79 \mathrm{e}-02$ & $1.93 \mathrm{e}-02$ & $1.69 \mathrm{e}-02$ \\
\hline
\end{tabular}

The results of the convergence analysis are summarized in Fig. 11 and quantified in Table 1, where we evaluate the global error with respect to both the $L^{2}(\Omega)$ - and the $H^{1}(\Omega)$-norm. The step size $h$ characterizing $\mathcal{T}_{h}$ is gradually halved, starting from the value 0.1 ; conversely, the modal index $m$ is doubled, starting from a single mode. For $h$ small enough, modal approximation slightly outperforms the expected convergence rates (error reduction factors are about 5 and 2.5 versus the expected 4 and 2, respectively). This is most likely related to the regularity of the solution. For the largest values of $h$, we have a slight stagnation of the error, in particular with respect to the $H^{1}(\Omega)$-norm. The error dependence on $h$ is evident only for high values of $m$, as the modal error dominates.

Neumann/Neumann Data We check the results of Theorem 5. For this purpose, we replace in (36) the conditions on $\Gamma_{u p}$ and $\Gamma_{\text {down }}$ with a homogeneous Neumann data, while preserving the condition on $\Gamma_{i n}$ and the free-flux condition on $\Gamma_{\text {out }}$. Thus, the exact solution is $u_{e x}=$ $y^{2}(1-y)^{2} \exp \left(\sin \left(20 y^{3}(1-y)^{2}(x-1)^{2}\right)\right)$. The Theorem 5 predicts order 4 and 3 with respect to the $L^{2}(\Omega)$ - and the $H^{1}(\Omega)$-norm, respectively. In Fig. 12 we provide the plot of 

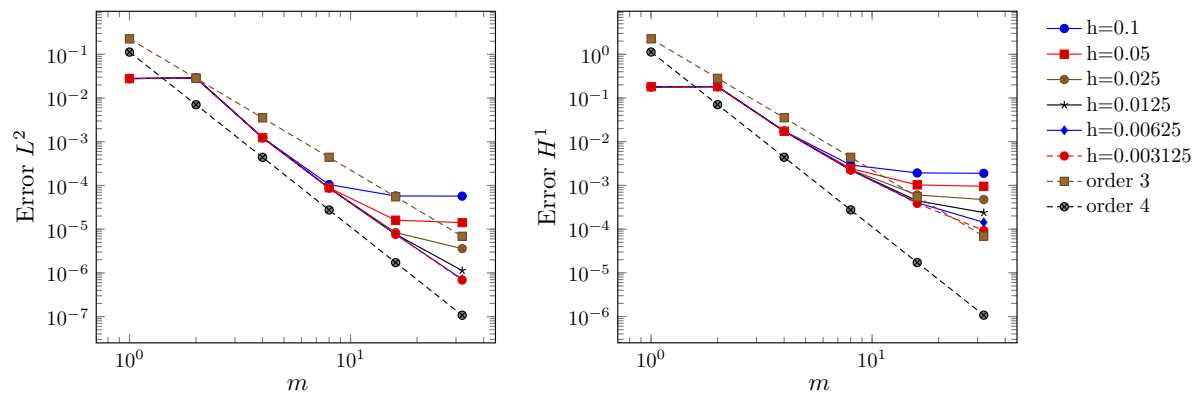

Fig. 12 2D convergence analysis, Neumann/Neumann data: global error with respect to the $L^{2}(\Omega)$-norm (left) and $H^{1}(\Omega)$-norm (right), for different discretization steps
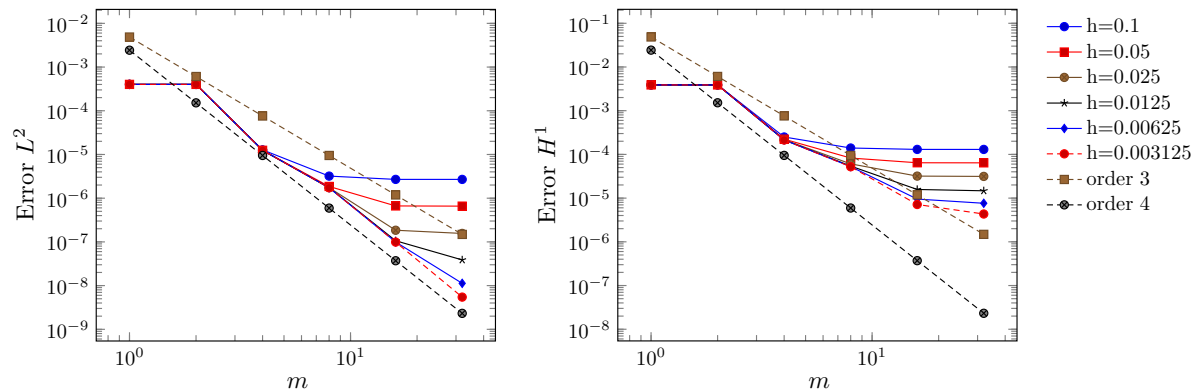

Fig. $132 \mathrm{D}$ convergence analysis, compatible data: global error with respect to the $L^{2}(\Omega)$-norm (left) and $H^{1}(\Omega)$-norm (right), for different discretization steps

the global error as a function of $m$ and for decreasing values of $h$. The plots associated with different mesh sizes are perfectly overlapped until 16 educated modes are used. The error stagnates except for the smallest values of $h$, showing a dominance of the finite element discretization error. For $h=0.0125,0.00625,0.003125$, the $L^{2}(\Omega)$-norm of the error shows the expected order of convergence, while the choices $h=0.00625, h=0.003125$ show the rate predicted by Theorem 5 for the $H^{1}(\Omega)$-norm. Finally, as for the Dirichlet/Robin data, the $H^{1}(\Omega)$-norm is less sensitive to the step size $h$.

Compatible Data We solve problem (36) by assigning homogeneous Dirichlet boundary conditions on $\Gamma_{u p} \cup \Gamma_{\text {down }}$. Thus, the exact solution reads $u_{e x}=y^{4}(1-y)^{4} e^{x}(x-1)^{2}$. This function satisfies compatible boundary conditions. In particular, the Laplacian of $u$ is identically equal to zero on $\Gamma_{u p}$ and $\Gamma_{d o w n}$, so that we expect a superconvergent trend when evaluating the global error with respect to the $L^{2}(\Omega)$ - and the $H^{1}(\Omega)$-norm, consistently with the results in Theorem 4 . This behavior is confirmed by Fig. 13. The convergence rate for the $L^{2}(\Omega)$-norm is about 4 for $h$ sufficiently small, as stated in (32). Concerning the $H^{1}(\Omega)$-norm, the finite element error induces a stagnation, preventing to neatly appreciate the expected modal error convergence rate.

\subsubsection{D Analysis}

Dirichlet Data We solve problem (1) on $\Omega=(0,0.2) \times(0,0.1)^{2}$, with $\mu=1, \boldsymbol{\beta}=(5,1,1)^{T}$ and $\sigma=3$. We impose homogeneous Dirichlet data on the whole $\Gamma_{w}$, while we assign a 

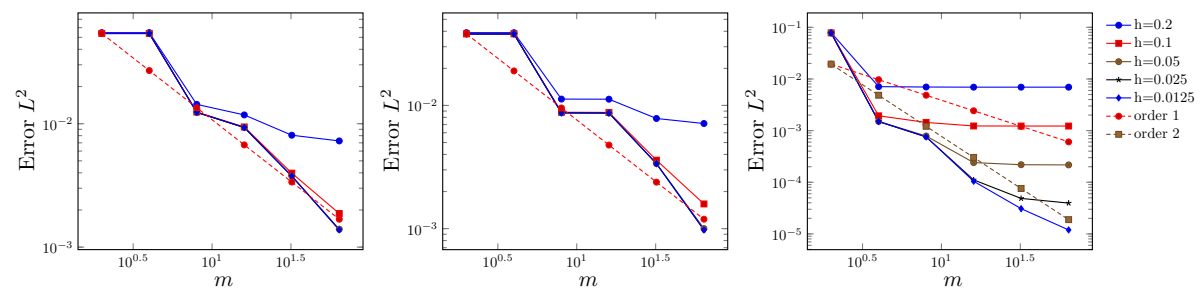

Fig. $143 \mathrm{D}$ convergence analysis: global error with respect to the $L^{2}(\Omega)$ - and $H^{1}(\Omega)$-norm for different discretization steps, in the presence of Dirichlet (left), Dirichlet/Robin (center) and Robin (right) data

Dirichlet data on $\Gamma_{1}$ and a homogeneous Neumann boundary condition on $\Gamma_{2}$. In particular, we select the source term and the Dirichlet condition on $\Gamma_{1}$ so that the exact solution is $u_{e x}(x, y, z)=10^{7} y(0.1-y) z(0.1-z)(x-0.2)^{2} \exp \left(2 y z(0.2-x)^{2}\right)$. As for the $2 \mathrm{D}$ analysis, we make different choices for the (uniform) spacing step along the supporting fiber $\Omega_{1 D}$, and then, for each selected $h$, we gradually increase the number of modal functions. Figure 14, left shows the trend of the global error for five choices of $h$. The modal order of convergence predicted for the $L^{2}(\Omega)$-norm by the theory in Sect. 4 is 1 . This is what we infer from the results in the figure, when the finite element error does not dominate.

Dirichlet/Robin Data We solve the standard Poisson problem on the cube $\Omega=(0,0.1)^{3}$, by assigning a homogeneous Dirichlet data on the upper and on the lower faces, $\Gamma_{u p}$ and $\Gamma_{d o w n}$, and the homogeneous Robin condition $\nabla u \cdot \mathbf{n}+3.345 u=0$ on $\Gamma_{w}$. The source term is selected such that the exact solution is $u_{e x}(x, y, z)=10^{5}(0.1-x)^{2} z(0.1-z) \exp \left(70 y^{2} /(x z+1)-\right.$ $\left.140 y^{3} /(0.3(x z+1))-3.345(0.1-2 y)^{2} /(0.4 \mu)\right)$. As shown in Fig. 14, center the $L^{2}(\Omega)$ norm of the global error exhibits a rate very similar to the one characterizing the fully Dirichlet case, showing how the e-HiMod approach does successfully extend the results derived for the Dirichlet case. In particular, for $m$ sufficiently large and for $h$ small enough, we obtain the expected rate of convergence, with a slight superconvergence for $h=0.025$ and $h=0.0125$.

Robin Data We modify the previous test case by assigning now a full Robin boundary condition $\nabla u \cdot \mathbf{n}+4.456 u=0$ on the entire surface $\partial \Omega$. We observe more sensitivity to the selected step size $h$ with respect to the previous choices of boundary conditions (compare the panel in Fig. 14, right with the two others). To check the modal convergence, we analyze the plot associated with $h=0.0125$ which essentially exhibit the expected linear rate, with a slight superconvergent trend. This can be likely justified by the fact that Robin conditions here quantitatively approaches Neumann conditions, which yield superconvergence, as for Theorem 5.

\subsection{Non Rectangular Geometries}

We conclude the numerical assessment of this section by analyzing the robustness of the e-HiMod approach on non-rectangular geometries.

\subsubsection{The 2D Backward Facing Step (2DBFS)}

We identify the computational domain $\Omega$ in (1) with the L-shaped portion $\Omega_{q} \backslash \Omega_{l}$ of the Cartesian plane, being $\Omega_{q}=(0,2) \times(-1,1)$ and $\Omega_{l}=(0,1) \times(-1,0)$. Moreover, we distinguish the following portions of the boundary $\partial \Omega: \Gamma_{\text {in }}=\{0\} \times[0,1]$ and $\Gamma_{\text {out }}=$ 

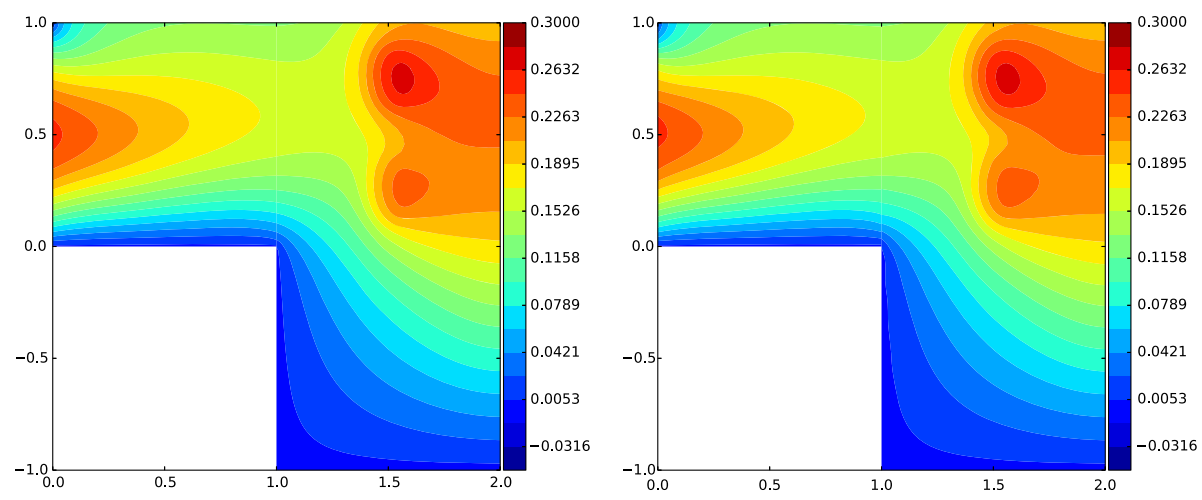

Fig. 15 2D backward facing step: reference finite element approximation (left); piecewise e-HiMod $(8,20)$ reduced solution (right)

$\{2\} \times[-1,1]$ coinciding with the inlet and the outlet, respectively; $\Gamma_{u p}=[0,2] \times\{1\}$ and $\Gamma_{\text {down }}=[1,2] \times\{-1\} \cup\{1\} \times[-1,0]$ to denote the upper and lower portion of the boundary, respectively. On this domain we solve the advection-diffusion problem

$$
\begin{cases}-\Delta u+\boldsymbol{\beta} \cdot \nabla u=f & \text { in } \Omega \\ u=y(1-y) & \text { on } \Gamma_{\text {in }} \\ \nabla u \cdot \mathbf{n}=0 \text { on } \Gamma_{\text {out }}, & \nabla u \cdot \mathbf{n}+u=0 \text { on } \Gamma_{u p} \\ u=0 & \text { on } \Gamma_{\text {down }},\end{cases}
$$

where the source term is $f(x, y)=10 \chi_{G_{1} \cup G_{2}}(x, y)$, with $G_{1}=\left\{(x, y) \in \Omega:(x-1.5)^{2}+\right.$ $\left.0.4(y-0.25)^{2} \leq 0.01\right\}$ and $G_{2}=\left\{(x, y) \in \Omega:(x-1.5)^{2}+0.4(y-0.75)^{2} \leq 0.01\right\}$, while the advective field $\boldsymbol{\beta}$ is the solution to the Stokes problem

$$
\begin{cases}-\nabla \cdot \mathbf{T}(\boldsymbol{\beta}, p)=0, \quad \nabla \cdot \boldsymbol{\beta}=0 & \text { in } \Omega \\ \mathbf{T}(\boldsymbol{\beta}, p) \mathbf{n}=5 \mathbf{n} \text { on } \Gamma_{\text {in }}, & \mathbf{T}(\boldsymbol{\beta}, p) \mathbf{n}=\mathbf{0} \text { on } \Gamma_{\text {out }} \\ \boldsymbol{\beta}=\mathbf{0} & \text { on } \Gamma_{w}=\Gamma_{\text {up }} \cup \Gamma_{\text {down }},\end{cases}
$$

with $\mathbf{T}=v\left(\nabla \boldsymbol{\beta}+\nabla \boldsymbol{\beta}^{T}\right)-p I$ the stress rate tensor depending on the velocity $\boldsymbol{\beta}$ and on the pressure $p$, being $v>0$ the kinematic viscosity and $I$ the identity matrix. Figure 15, left shows the contour plot of a reference solution to problem (37) computed via linear finite elements on a structured uniform mesh of sizes $h_{x}=h_{y}=0.01$. The same mesh has been employed to compute $\boldsymbol{\beta}$ via a standard two-dimensional P2-P1 finite element solver, so that the convective field is piecewise quadratic. Its values in the quadrature nodes during the assembly are retrieved accordingly.

To approximate problem (37) via an e-HiMod procedure, we resort to the piecewise HiMod formulation proposed in [27] to tackle phenomena characterized by a different smoothness in distinct areas of the domain. The idea simply consists in employing a different number of modes in these areas according to the local transverse features of the solution. Following this approach, we split $\Omega$ into the two subdomains $\Omega_{1}=(0,1)^{2}$ and $\Omega_{2}=(1,2) \times(-1,1)$, and we apply, on each of them, the standard e-HiMod reduction. Then, the two reduced models are merged via a domain decomposition iterative scheme. In particular, we adopt a relaxed Dirichlet/Neumann scheme in correspondence with the interface $\Gamma=\{1\} \times(0,1)$ between 

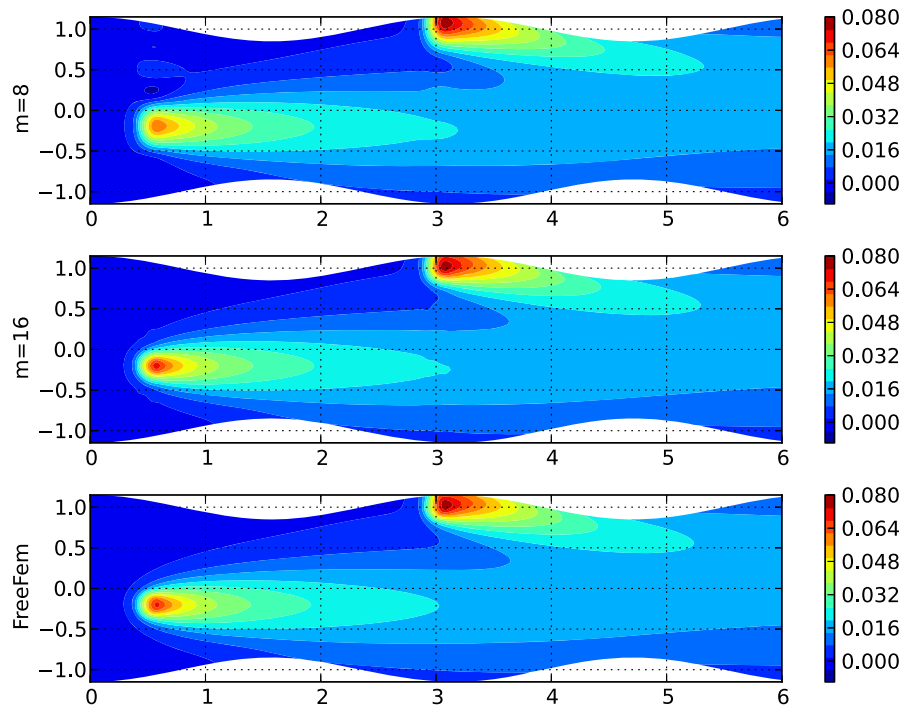

Fig. 16 e-HiMod $(m)$ versus finite elements on a wavy channel geometry. From the top to the bottom: e-HiMod(8), e-HiMod(16), finite elements

$\Omega_{1}$ and $\Omega_{2}$. We use 8 and 20 educated modal functions on $\Omega_{1}$ and $\Omega_{2}$, respectively while introducing a uniform subdivision along the supporting fiber $\Omega_{1 D}$ of step-length $h_{x}=0.01$.

The corresponding reduced solution, denoted by e-HiMod$(8,20)$, is shown in Fig. 15, right. It compares excellently with the reference finite element approximation, yet with a significant reduction of dof, even in capturing the transverse dynamics induced by the geometry that would be dropped in a purely one-dimensional model.

\subsubsection{A Wavy Channel Geometry}

Fig. 16 displays the results of an ADR problem with Robin boundary conditions on the lateral boundaries, as in the previous section, in a wavy channel domain, to mimic Oxygen dynamics in a Bellhouse oxygenator (see also [27]). The modal approximation is obtained by e-HiMod. The figure compares the results with a fine-mesh finite element simulation, showing how the main features of the dynamics are captured with just 8 modes, while with $m=16$ case displays an excellent overlapping with the reference solution obtained with many more degrees of freedom.

\subsubsection{The 3D Backward Facing Step (3DBFS)}

We solve on the $3 \mathrm{D}$ domain $\Omega=\Omega_{Q} \backslash \Omega_{L}$, with $\Omega_{Q}=(0,2) \times(0,1) \times(0,2)$ and $\Omega_{L}=(0,1)^{2} \times(1,2)$, the advection-diffusion problem

$$
\begin{cases}-\Delta u+\boldsymbol{\beta} \cdot \nabla u=f & \text { in } \Omega \\ u=g \text { on } \Gamma_{\text {in }}, & \nabla u \cdot \mathbf{n}=0 \text { on } \Gamma_{\text {out }} u=0 \text { on } \Gamma_{w},\end{cases}
$$

where, for the sake of simplicity, we select a constant field, $\boldsymbol{\beta}=(20,0,7)^{T}$, and with $\Gamma_{1}=\{x=0\} \times(0,1)^{2}, \Gamma_{2}=\{x=2\} \times(0,1) \times(0,2), \Gamma_{w}=\partial \Omega \backslash\left(\Gamma_{\text {in }} \cup \Gamma_{\text {out }}\right)$. The 

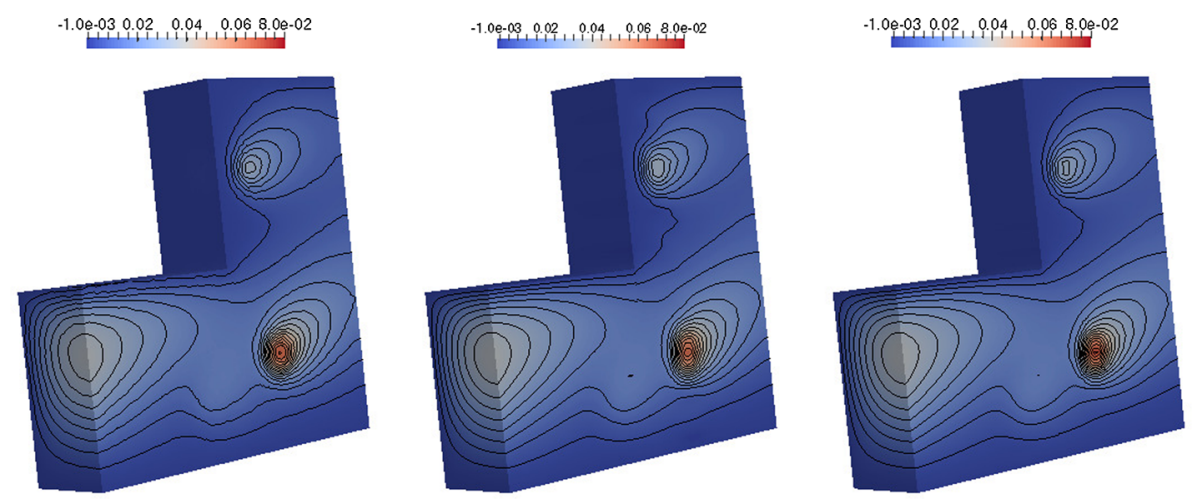

Fig. 17 3DBFS, Dirichlet data: reference finite element approximation (left); HiMod(100) (center) and HiMod(200) (right) reduced solutions

forcing term is localized in correspondence with three spherical regions, being $f(x, y, z)=$ $20 \chi_{S_{1} \cup S_{2} \cup S_{3}}(x, y, z)$, with $S_{1}=\left\{(x, y, z) \in \Omega:(x-0.7)^{2}+(y-0.3)^{2}+(z-0.3)^{2} \leq\right.$ $0.1\}, S_{2}=\left\{(x, y, z) \in \Omega:(x-1.3)^{2}+(y-0.5)^{2}+(z-0.5)^{2} \leq 0.1\right\}, S_{3}=\{(x, y, z) \in$ $\left.\Omega:(x-1.2)^{2}+(y-0.6)^{2}+(z-1.5)^{2} \leq 0.1\right\}$. Figure 17, left shows the contour plot of the reference 3D linear finite element approximation computed on a structured mesh of uniform sizes, $h_{x}=h_{y}=h_{z}=1 / 30$. In particular, the plot refers to the transverse section at $y=0.5$. It is evident the effect of the Dirichlet data at the inflow, of the field $\boldsymbol{\beta}$ and of the sources, even though the selected section weakly highlights the presence of $S_{1}$, while emphasizing the effect of $S_{2}$ and $S_{3}$.

Concerning the HiMod reduction, we resort to a piecewise hierarchical model reduction applied to the subdomains $\Omega_{1}=(0,1)^{3}$ and $\Omega_{2}=(1,2) \times(0,1) \times(0,2)$. Homogeneous Dirichlet data on the entire lateral surface lead us to consider standard sinusoidal functions as modes. A uniform one-dimensional discretization of step size $h=1 / 30$ is employed along the supporting fiber $\Omega_{1 D}$. We analyze two different configurations, with 100 and 200 modes in both the subdomains, respectively corresponding to the first 100 and 200 eigenfunctions of the Laplace operator computed with the ESA.. The relaxed Dirichlet/Neumann scheme converges after a few iterations and provides the approximations in Fig. 17, center $(m=100)$ and right $(m=200)$. The HiMod(100) solution is comparable with the reference one in Fig. 17, left despite a lack of accuracy can be appreciated along the edge $E=\{x=1\} \times(0,1) \times\{z=1\}$. The matching of the two reduced solutions along $E$ is a challenging task. In fact, the modal functions on $\Omega_{1}$ are identically equal to zero on $(0,1)^{2} \times\{z=1\}$ due to the Dirichlet data, whilst the modes involved in $\Omega_{2}$ are free to assume any value across $(1,2) \times(0,1) \times\{z=1\}$. As expected, the mismatch between the two approximations diminishes when we move away from $E$, since no boundary data constrains the projection of a HiMod basis onto the other one. This effect fades away when considering the finer approximation, $\operatorname{HiMod}(200)$.

To assess the performance of the e-HiMod procedure, we modify the boundary data assigned on the faces $F_{B}=(0,2) \times(0,1) \times\{z=0\}$ and $F_{T}=(1,2) \times(0,1) \times\{z=2\}$. Here we enforce now the Robin data $\nabla u \cdot \mathbf{n}+4 u=0$. We also move the location of the sources $S_{2}$ and $S_{3}$ by centering them at $(1.1,0.5,0.1)$ and $(1.3,0.5,1.5)$, respectively. The resulting dynamics is non-trivial on $F_{B}$. We computed the finite element solution for a different number of dof. In particular, here for brevity, we compare the standard and the e-HiMod approach for a similar number of dof, namely 6547 for finite elements and 6100 for 

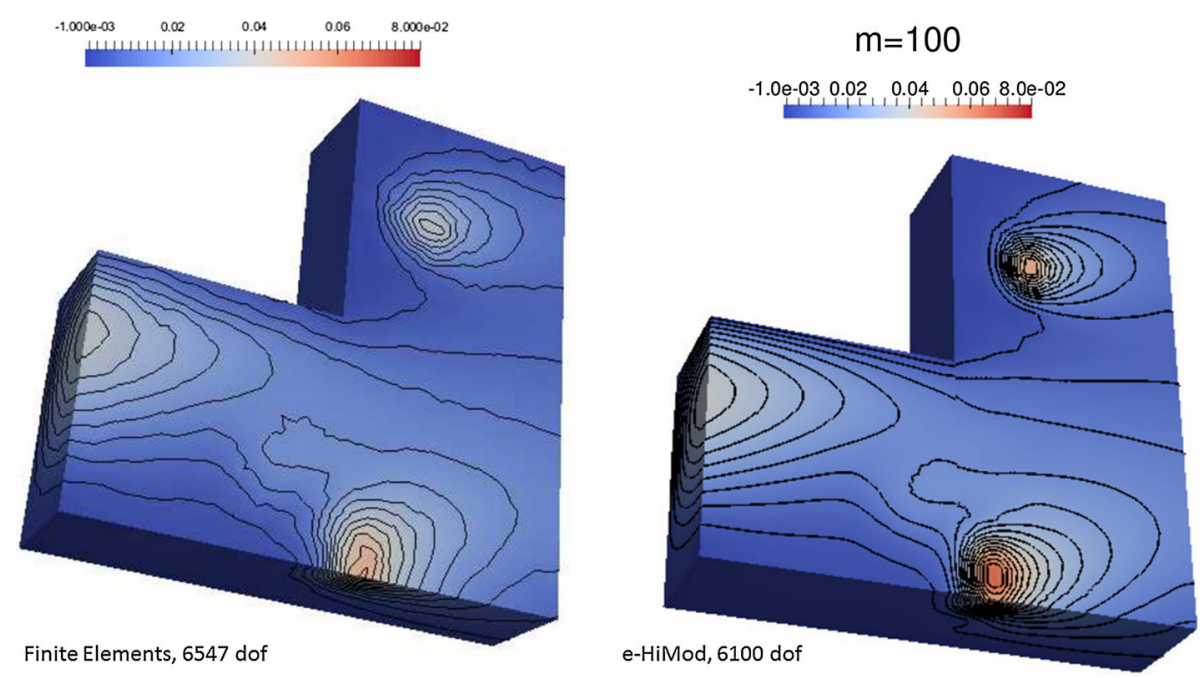

Fig. 18 3DBFS, Dirichlet/Robin data: coarse finite element solution versus e-HiMod with a comparable number of dof

e-HiMod, respectively. The latter number corresponds to $m=100$, i.e. we constructed the basis with the first 100 eigenfunctions of $\mathcal{L}_{S}$. The comparison outlines how, for a comparable number of dof, e-HiMod outperforms standard approaches (Fig. 18). While refinements of the two discretizations will clearly converge to the same solution, e-HiMod constantly maintains a better management of the discretization errors for the diversification of the discretization in the different directions, as already demonstrated in [22] on patient-specific coronary arteries for homogeneous Dirichlet conditions.

\section{Conclusions and Perspectives}

Several details of the HiMod reduction procedure need to be still addressed. In this paper we have extensively considered the problem of assigning general boundary conditions on the wall of pipes. This is a crucial issue in view of practical applications of HiMod, for instance, to fluid-structure interaction problems, where the effect of the structure on the fluid can be modeled by Robin boundary conditions on the vessel boundaries [4]. We demonstrated that the construction of customized functions based on the solution of SLE problems provides an effective approach for automatically incorporating general boundary conditions with the same performances previously obtained for Dirichlet conditions. Theory has been worked out, with reference to the SLE approximation theory. We also detected and explained super-convergent cases, depending on the type of boundary conditions and on the regularity of the solution. Results in Sect. 5 are intended to provide a proof of concept of the e-HiMod procedure and to corroborate the proposed theory. We stress again that e-HiMod is not mainly intended to compete with standard three-dimensional solver, rather to give a method for modulating the accuracy for the secondary transverse dynamics, so to be able of covering networks of pipes by improving simple one-dimensional models. Indeed, the numerical assessment shows that e-HiMod can work on non-trivial geometries (as already demonstrated for Dirichlet conditions $[6,22]$ ) yet approaching the full solution via a "psychological" one-dimensional 
framework. An appropriate selection of the modes $m$ can attain the right trade-off for working on networks. For test cases on pipe domains in cylindrical coordinates, we refer to [17].

Following up the contributions mentioned above, we plan to apply the e-HiMod procedure to more complex problems, including the incompressible Navier-Stokes equations in both rectilinear and curved cylindrical pipes, to be applied to simplified models of the human circulation, in the spirit of $[6,22]$. Concerning curved geometries, after a first contribution in [26], curved geometries have been considered more recently in [28] — where isogeometric analysis replaces the finite element discretization along the mainstream—and in [22] with a Legendre polynomial approximation of transverse dynamics (called Transversally Enriched Pipe Element Method). Bifurcations are partially worked out in [6]. It is worth reminding that one-dimensional networks in fluid dynamics are currently the only viable option, for instance, for mathematical modeling of the human circulation over a large number of vessels (see [7] with more than 2000 arteries included). The e-HiMod approach candidates to work out similar problems with more accuracy, possibly adapted to specific regions of interest for the rapid and reliable simulation of blood in the circulation.

Finally, the computational advantages of the one-dimensional coupled pattern of the linear system characterizing the e-HiMod procedure needs to be explored as well. This is expected to further improve the computational advantages of the HiMod methodology.

Acknowledgements The second and the third authors acknowledge the support of NSF Grant DMS-1419060 "Hierarchical Model Reduction Techniques for Incompressible Fluid-Dynamics and Fluid-Structure Interaction Problems" for this research. The authors wish to thank also Prof. Sandro Salsa for fruitful discussions concerning the analysis of the method, and Pablo J. Blanco for hosting the first author for one month at Laboratório Nacional de Computacao Científica in Petrópolis, Brazil. This article has been partially supported by Istituto Nazionale di Alta Matematica "Francesco Severi" (IT) Project (GNCS2017 Metodi numerici avanzati combinati con tecniche di riduzione computazionale per PDEs parametrizzate ed applicazioni).

\section{References}

1. Aletti, M.C.: Educated basis for hierarchical model reduction in $2 \mathrm{D}$ and 3D. Master thesis, Politecnico di Milano, Italy (a.y. 2012-2013)

2. Aletti, M., Bortolossi, A., Perotto, S., Veneziani, A.: One-dimensional surrogate models for advectiondiffusion problems. In: Abdulle, A., Deparis, S., Kressner, D., Nobile, F., Picasso, M. (eds.) Numerical Mathematics and Advanced Applications, Lecture Notes Computer Science and Engineering, vol. 103, pp. 447-456. Springer (2015)

3. Azaiez, M., Shen, J., Xu, C., Zhuang, Q.: A Laguerre-Legendre spectral method for the Stokes problem in a semi-infinite channel. SIAM J. Numer. Anal. 47(1), 271-292 (2009)

4. Badia, S., Nobile, F., Vergara, C.: Fluidstructure partitioned procedures based on Robin transmission conditions. J. Comput. Phys. 227(14), 7027-7051 (2008)

5. Barone, A.: Parallel and multilevel techniques for hierarchical model reduction, Master thesis, Politecnico di Milano, Italy (a.y. 2013-2014)

6. Blanco, P.J., Mansilla Alvarez, L.A., Feijoo, R.A.: Hybrid element-based approximation for the NavierStokes equations in pipe-like domains. Comput. Methods Appl. Mech. Eng. 283, 971-993 (2015)

7. Blanco, P.J., Watanabe, S.M., Passos, M.A.R.F., Lemos, P.A., Feijoo, R.A.: An anatomically detailed arterial network model for one-dimensional computational hemodynamic. IEEE Trans. Biomed. Eng. 62, 736-753 (2015)

8. Boyd, J.P.: Chebyshev and Fourier Spectral Methods. Dover, Mineola (2001)

9. Canuto, C., Hussaini, Y., Quarteroni, A., Zang, T.A.: Spectral Methods: Fundamentals in Single Domains. Springer, Berlin (2006)

10. Canuto, C., Hussaini, Y., Quarteroni, A., Zang, T.A.: Spectral Methods: Evolution to Complex Geometries and Applications to Fluid Dynamics. Springer, Berlin (2007)

11. Canuto, C., Maday, Y., Quarteroni, A.: Analysis of the combined finite element and Fourier interpolation. Numer. Math. 39(2), 205-220 (1982) 
12. Canuto, C., Maday, Y., Quarteroni, A.: Combined finite element and spectral approximation of the NavierStokes equations. Numer. Math. 44(2), 201-217 (1984)

13. Ern, A., Guermond, J.-L.: Theory and Practice of Finite Elements. Applied Mathematical Sciences, vol. 159. Springer, New York (2004)

14. Ern, A., Perotto, S., Veneziani, A.: Hierarchical model reduction for advection-diffusion-reaction problems. In: Kunisch, K., Of, G., Steinbach, O. (eds.) Numerical Mathematics and Advanced Applications, pp. 703-710. Springer, Berlin (2008)

15. Estrada, E.: The Structure of Complex Networks: Theory and Applications. Oxford University Press, Oxford (2011)

16. Formaggia, L., Quarteroni, A., Veneziani, A.: Multiscale models for the vascular system. In: Formaggia, L., Quarteroni, A., Veneziani, A. (eds.) Cardiovascular Mathematics, pp. 395-446. Springer, Berlin (2009)

17. Guzzetti, S., Perotto, S., Veneziani, A.: Hierarchical model reduction in cylindrical domains. MOX report 51/2016, Dipartimento di Matematica, Politecnico di Milano

18. Hecth, F.: New developement in Freefem++. J. Numer. Math. 20(3-4), 251-265 (2012)

19. Karniadakis, G., Sherwin, S.: Spectral/hp Element Methods for Computational Fluid Dynamics. Oxford University Press, Oxford (2013)

20. Landau, L.: Monotonicity and bounds on Bessel functions. Mathematical physics and quantum field theory. Electron. J. Differ. Equ. Conf. 04, 147-154 (2000)

21. Lions, J.L., Magenes, E.: Non Homogeneous Boundary Value Problems and Applications. Springer, Berlin (1972)

22. Mansilla Alvarez, L.A., Blanco, P.J., Feijoo, R.A., Bulant, C.A., Dari, E.A., Veneziani, A.: Transversally enriched pipe element method (TEPEM). An effective numerical approach for blood flow modeling. Int. J. Numer. Methods Biomed. Eng. 33, e2808 (2017)

23. Ohlberger, M., Smetana, K.: A dimensional reduction approach based on the application of reduced basis methods in the framework of hierarchical model reduction. SIAM J. Sci. Comput. 36(2), A714-A736 (2014)

24. Osiadacz, A.: Simulation and Analysis of Gas Networks. Gulf Publishing Company, Houston (1987)

25. Peiró, J., Veneziani, A.: Reduced models for the cardiovascular system. In: Formaggia, L., Quarteroni, A., Veneziani, A. (eds.) Cardiovascular Mathematics, pp. 347-394. Springer, Berlin (2009)

26. Perotto, S.: Hierarchical model (Hi-Mod) reduction in non-rectilinear domains. In: Erhel, J., Gander, M., Halpern, L., Pichot, G., Sassi, T., Widlund, O. (eds.) Domain Decomposition Methods in Science and Engineering, Lecture Notes in Computer Science and Engineering, vol. 98, pp. 477-485. Springer, Cham (2014)

27. Perotto, S., Ern, A., Veneziani, A.: Hierarchical local model reduction for elliptic problems: a domain decomposition approach. Multiscale Model. Simul. 8(4), 1102-1127 (2010)

28. Perotto, S., Reali, A., Rusconi, P., Veneziani, A.: HIGAMod: A Hierarchical IsoGeometric Approach for MODel reduction in curved pipes. Comput. Fluids 142, 121-129 (2016)

29. Perotto, S., Veneziani, A.: Coupled model and grid adaptivity in hierarchical reduction of elliptic problems. J. Sci. Comput. 60(3), 505-536 (2014)

30. Perotto, S., Zilio, A.: Hierarchical model reduction: three different approaches. In: Cangiani, A., Davidchack, R.L., Georgoulis, E., Gorban, A.N., Levesley, J., Tretyakov, M.V. (eds.) Numerical Mathematics and Advanced Applications, pp. 851-859. Springer, Berlin (2013)

31. Perotto, S., Zilio, A.: Space-time adaptive hierarchical model reduction for parabolic equations. Adv. Model. Simul. Eng. Sci. 2(25), 1-45 (2015)

32. Pinchover, Y., Rubinstein, J.: An Introduction to Partial Differential Equations. Cambridge University Press, Cambridge (2005)

33. Quarteroni, A., Veneziani, A., Vergara, C.: Geometric multiscale modeling of the circulatory system between theory and practice. Comput. Methods Appl. Mech. Eng. 302, 193-252 (2016)

34. Salsa, S.: Partial Differential Equations in Action. Springer, Milan (2015)

35. Toselli, A., Widlund, O.: Domain Decomposition Methods-Algorithms and Theory. Springer, Berlin (2006)

36. Vogelius, M., Babuška, I.: On a dimensional reduction method. I. The optimal selection of basis functions. Math. Comput. 37, 31-46 (1981)

37. Vogelius, M., Babuška, I.: On a dimensional reduction method. II. Some approximation-theoretic results. Math. Comput. 37, 47-68 (1981)

38. Vogelius, M., Babuška, I.: On a dimensional reduction method. III. A posteriori error estimation and an adaptive approach. Math. Comput. 37, 361-384 (1981)

39. Zielinski, L.: Asymptotic distribution of eigenvalues for elliptic boundary value problems. Asymptot. Anal. 16(3), 181-201 (1998) 